Article

\title{
Perturbative Peculiarities of Quantum Field Theories at High Temperatures
}

\author{
Ingolf Bischer ${ }^{1}$, Thierry Grandou ${ }^{2, *}$ and Ralf Hofmann ${ }^{3,4}$ \\ 1 Max-Planck-Institut für Kernphysik, Postfach 103980, D-69029 Heidelberg, Germany; \\ bischer@mpi-hd.mpg.de \\ 2 University Cote d'Azur, CNRS, INPHYNI, 1361 routes des Lucioles, 06560 Valbonne, France \\ 3 Institut für Theoretische Physik, Universität Heidelberg, Philosophenweg 16, 69120 Heidelberg, Germany; \\ r.hofmann@thphys.uni-heidelberg.de \\ 4 Institut for Photon Science and Sychrotron Radiation, Karlsruher Institut für Technologie, \\ Hermann-von-Helmholtz-Platz 1, D-76344 Eggenstein-Leopoldshafen, Germany \\ * Correspondence: thierry.grandou@inphyni.cnrs.fr
}

Received: 13 February 2019; Accepted: 6 March 2019; Published: 14 March 2019

\begin{abstract}
Revisiting the fast fermion damping rate calculation in a thermalized QED and/or QCD plasma in thermal equilibrium at four-loop order, focus is put on a peculiar perturbative structure which has no equivalent at zero-temperature. Not surprisingly, and in agreement with previous $C^{\star}$-algebraic analyses, this structure renders the use of thermal perturbation theory more than questionable.
\end{abstract}

Keywords: thermal perturbative QCD; damping rates

\section{Introduction}

As quoted long ago by Pisarski [1], "It's really surprising how difficult it is to calculate damping rates in hot gauge field theories".

Even though a so-called Resummation Program of Hard Thermal Loops (that are leading order thermal fluctuations at high temperature, hereafter denoted by $R P$ ) has been devised to obtain gauge-invariant complete results to damping rates (and has succeeded to some extent [2-4]), serious difficulties have constantly bounced back and forth within the infrared sector of hot gauge field theories.

Literature testifies of two major obstructions to the Resummation Program that have become textbook material [5]. The first one was discovered about the same time as the Resummation Program itself [6], while using the latter in the situation to be described in the next section. The second obstruction was discovered a few years later, while using the Resummation Program to evaluate the soft photon emission rate out of a Quark-Gluon Plasma in thermal equilibrium [7]. In both situations, the hot gauge theories infrared sector was recognized to be at the origin of two singular results.

In the latter case, the singular result reads $(D=4+2 \varepsilon)$,

$$
\frac{C^{s t}}{\varepsilon} \int \frac{d^{4} P}{(2 \pi)^{4}} \delta(\widehat{Q} \cdot P)\left(1-2 n_{F}\left(p_{0}, T\right)\right) \sum_{s= \pm 1, V=P, P^{\prime}} \pi\left(1-s \frac{v_{0}}{v}\right) \beta_{s}(V),
$$

where $\beta_{s}(V)$ is the space-like part of the fermionic spectral densities, and $V=P, P^{\prime}$ with $P^{\prime}=P+$ $Q$, [5]. The explicit Lorentz invariance breaking appears in the statistical factor $n_{F}\left(p_{0}, T\right)$ due to its dependence on $T$, which associates with a preferred rest frame. In effect, in the context of quantum field theories, temperature at thermodynamical equilibrium can be implemented in a covariant way by means of a Lorentz 4-vector, $\beta$, of squared norm $\beta^{2}=(1 / T)^{2}$. (We work in units where $k_{B}$, Boltzmann's 
constant, is set equal to unity.) Only in the reference frame, where the plasma is at rest and in thermal equlibrium such that $u=(1, \overrightarrow{0})$ ( $u$ the 4 -velocity of the plasma) does the invariant $(u \cdot \beta)^{-1}=T$ allow for the interpretation of a temperature. The singularity in Equation (1) is due to the following double entwined angular integrals. With $\widehat{K}, \widehat{K}^{\prime}$, the two light-like 4 -vectors $(1, \hat{k})$ and $\left(1, \hat{k}^{\prime}\right)$,

$$
W\left(P, P^{\prime}\right)=\sum_{s, s^{\prime}= \pm} \int \frac{\mathrm{d} \widehat{K}}{4 \pi} \int \frac{\mathrm{d} \widehat{K}^{\prime}}{4 \pi} \widehat{K} \cdot \widehat{K}^{\prime} \frac{\widehat{K} \cdot \widehat{P}_{s} \widehat{K}^{\prime} \cdot \widehat{P}_{s^{\prime}}^{\prime}+\widehat{K} \cdot \widehat{P}_{s^{\prime}}^{\prime} \widehat{K}^{\prime} \cdot \widehat{P_{s}}-\widehat{K} \cdot \widehat{K}^{\prime} \widehat{P}_{s} \cdot \widehat{P}_{s^{\prime}}^{\prime}}{(\widehat{K} \cdot P+i \epsilon)\left(\widehat{K} \cdot P^{\prime}+i \epsilon\right)\left(\widehat{K}^{\prime} \cdot P+i \epsilon\right)\left(\widehat{K}^{\prime} \cdot P^{\prime}+i \epsilon\right)},
$$

which, to our knowledge, computers cannot evaluate. An estimated singular behaviour of $W\left(P, P^{\prime}\right)$ was accordingly retained, giving rise to the singular result above, whereas a full, exact and cross-checked calculation of $W\left(P, P^{\prime}\right)$ displays a series of mass singularities of strengths $\varepsilon^{-1}$ and $\varepsilon^{-2}$, which exactly cancel out among themselves, leaving a regular result [8-10].

In the former case, the current revisitation of the problem will conclude that its singular result has been induly derived as well.

As will be stated in the conclusion of this analysis, it turns out that the issue of infrared singularities has long masked a more fundamental difficulty, which appears to be inherent to the perturbative approach and has been loosely identified within the context of $C^{\star}$-algebraic analyses [11]. Elaborating on this fundamental difficulty is the objective of the current paper, organized as follows.

Section 2 is a quick reminder of the historical fast fermion damping rate calculation in a thermalized quantum electrodynamics (QED) or quantum chromodynamics (QCD) plasmas. The notations to be used throughout the article, as well as the process basic diagram are given.

Section 3 accounts for the photon polarization tensor at two-loop order, or equivalently, fast muon damping rate at three loops. The leading order contributions are retained in the limit of $k_{\mu} / T \ll 1$ where $k_{\mu}$ is a component of the internal photonic line, $K$. This is essentially technical, with some overall detailed balance examples of infrared (mass) singularity compensations displayed. The important point is comprised within the simple Equation (55), which expresses the leading form of the photon polarization tensor at two-loop order.

Section 4 announces the point of the current paper, the peculiarity of the thermal case, by contrasting it to the customary $T=0$ situation, as an independent resummation of leading fluctuations occurring at momentum scale $e^{2} T$ is shown to be necessary.

While it is believed that the ultrasoft scale is terminal, receiving contributions from higher number of loop diagrams, a three-loop evaluation of the photon polarization tensor appears to display an interplay of thermal and vacuum fluctuations leading to contributions of dominant order and calling for another independent resummation along the internal photonic line. Revealed by a high enough number of loop calculation, these leading mixed fluctuations of the photonic internal line take place at momentum scale $e^{3} T$, are gauge invariant and contribute to the fast muon damping rate being on the same footing as the two first ones, at soft $(e T)$ and ultrasoft $\left(e^{2} T\right)$ momentum scales. This is what is dealt with in Section 5 .

Since only the contributions attached to the longitudinal degree of freedom of the internal photonic line were considered in the previous sections, the contributions of transverse degrees are examined in Section 6 so as to make sure that there is not a full annihilation of the contributions due to the longitudinal leading order mixed fluctuations.

Section 7 is a discussion of our results in the light of previous $C^{\star}$-algebraic analysis. The relevance of a perturbative approach bare or effective is questioned while possible non-perturbative alternative approaches are mentioned. Bare perturbation theory admits the same diagrammatic expansions as customary $T=0$ quantum field theories, but now subject to the $T \neq 0$-free field propagators and vertices [5]. 


\section{A Reminder of the Fast Muon Damping Rate Issue}

The energy loss of a heavy fermion, a muon for example, propagating through a QED plasma is considered. It is assumed that the heavy muon has mass $M$ much larger than the temperature $T$, and that, travelling through the plasma, it retains momenta $p \gg T$ and thus is not thermalised. In the plasma, electrons (positrons) are assumed to be massless, and, for the muon, energy loss is induced by Compton scattering off photons and Coulomb scattering off electrons and positrons of the plasma.

In the framework of real-time thermal perturbation theory of QED, at temperature $T$, the damping rate is related to the fermionic self energy $\Sigma(P)$ by the relation $[5,12]$,

$$
\gamma(E, \vec{p}, T)=-\frac{1}{4 E} \operatorname{Tr}\left((P+M) \operatorname{Im} \Sigma\left(p_{0}, \vec{p}, T\right)\right)_{\left.\right|_{p_{0}=E}} .
$$

The notation $K=\left(k_{0}, \vec{k}\right)$ will be used throughout. In order to get rid of inessential complications, it has been customary and convenient to assume the following ordering relations,

$$
E, p=|\vec{p}| \gg M \gg T \gg e T .
$$

In view of $p / \sqrt{p^{2}+M^{2}} \simeq 1$, in effect, Equation (3) allows one to take the high velocity limit $v=p / E=1$ in coincidence with the fermion mass shell limit, as in the massless case. We assume zero chemical potential and a high temperature, which is much bigger than the electron, positron or quark masses. One gets

$$
\gamma(E, \vec{p}, T)=-\frac{1}{4 E} \int \frac{\mathrm{d}^{4} K}{(2 \pi)^{3}} \delta_{+}\left(P^{\prime 2}-M^{2}\right) D_{\mu v}^{+}\left(k_{0}, \vec{k}, T\right) \operatorname{Tr}\left((\not P+M) \gamma^{\mu}\left(P^{\prime}+M\right) \gamma^{v}\right),
$$

where $P^{\prime}=P+K$, as depicted on Figure 1. In Equation (4), the $\delta_{+}$distribution is the muon cut propagator taken at $T=0$, since, as stated above, this real particle is not at thermal equilibrium with the QED plasma. The function $D_{\mu \nu}^{+}$instead is the thermalized cut photon propagator in Coulomb gauge. Whenever the $R P$ is used, quantities are denoted with a symbol ${ }^{\star}$, and one has

$$
{ }^{\star} D_{\mu v}^{+}\left(k_{0}, \vec{k}, T\right)=\left(1+n_{B}\left(k_{0}, T\right)\right)\left(\mathcal{P}_{\mu v}^{L}(K)^{\star} \rho_{L}\left(k_{0}, \vec{k}, T\right)+\mathcal{P}_{\mu v}^{T}(K)^{\star} \rho_{T}\left(k_{0}, \vec{k}, T\right)\right) .
$$

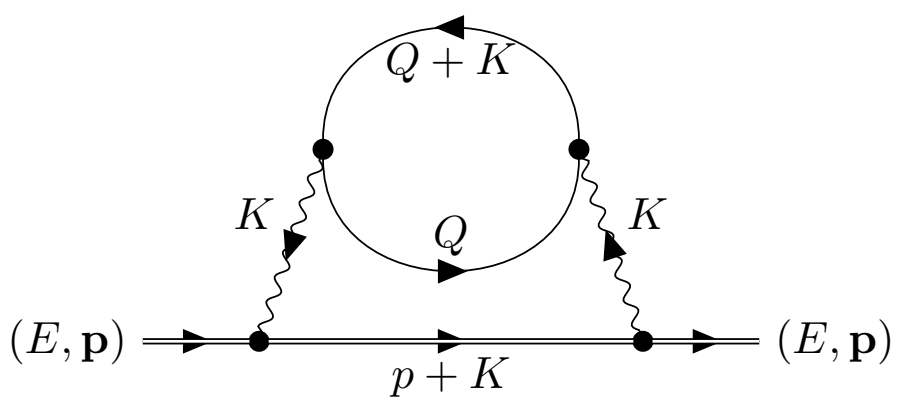

Figure 1. A two-loop contribution to $\gamma(E, \vec{p})$. The polarization tensor $\Pi^{(1)}\left(k_{0}, k\right)$ is the Hard Thermal Loop (HTL) one from Equation (12).

The operators $\mathcal{P}_{\mu \nu}^{L, T}$ are the $4 D$ - longitudinal and transverse projectors [5], and ${ }^{\star} \rho_{T, L}$ the transverse and longitudinal spectral densities of the effective propagator. Eventually, $n_{B}\left(k_{0}, T\right)$ is the ordinary Bose-Einstein statistical factor, but in the $R / A$ - real-time formalism being used [5], without absolute value prescription, for example, $n_{B}\left(p_{0}, T\right)=\frac{1}{e^{p_{0} / T}-1}$. 
Summing Equation (4) over the angles by taking advantage of the distribution $\delta_{+}$one gets, with $x=k_{0} / k$ and $k=|\vec{k}|$,

$$
{ }^{\star} \gamma(E, p, T)=\frac{e^{2}}{2 \pi v} \int k^{2} \mathrm{~d} k \int_{-v}^{+v} \frac{\mathrm{d} x}{2 \pi}\left(1+n_{B}(k x, T)\right)\left\{{ }^{\star} \rho_{L}(k x, k, T)+\left(v^{2}-x^{2}\right)^{\star} \rho_{T}(k x, k, T)\right\} .
$$

Next, since momentum $K$ is soft, on the order of $e T$, one expands the statistical factor and writes,

$$
{ }^{\star} \gamma(E, p, T)=\frac{e^{2}}{2 \pi v} \int k^{2} \mathrm{~d} k \int_{-v}^{+v} \frac{\mathrm{d} x}{2 \pi}\left(\frac{T}{k x}+\frac{1}{2}+\mathcal{O}\left(\frac{k x}{T}\right)\right)\left\{{ }^{\star} \rho_{L}(k x, k, T)+\left(v^{2}-x^{2}\right)^{\star} \rho_{T}(k x, k, T)\right\} .
$$

The most infrared dangerous piece of the Bose-Einstein factor expansion is the term $T / k x$ which is hereafter retained. Appealing to the energy sum rule,

$$
\int_{-1}^{+1} \frac{d x}{2 \pi} \frac{{ }^{\star} \rho_{T}(k x, k)}{x} \simeq \frac{1}{k^{2}}-\frac{3}{2 m^{2}}
$$

where $m$ is defined below, Equation (14), one obtains, out of the transverse part, the well known mass shell singular result,

$$
{ }^{\star} \gamma(E, p, T)_{\mid v=1}=\frac{e^{2} T}{2 \pi} \int_{k_{m}}^{k_{M}} \frac{\mathrm{d} k}{k}+\text { regular, } \quad k_{m}=\frac{\left|E-\sqrt{p^{2}+M^{2}}\right|}{2 v} \simeq \frac{E|1-v|}{2 v},
$$

where the logarithmic diverging behaviour by the fermion mass shell is manifest. The upper bound, $k_{M}$, is some conveniently choosen intermediate scale between the soft, $\mathcal{O}(e T)$, and hard $\mathcal{O}(T)$ scales, usually the scale of order $\sqrt{e} T$. The ${ }^{\star} \rho_{L}$ contribution, instead, is regular, the relevant sum rule being,

$$
\int_{-1}^{+1} \frac{\mathrm{d} x}{2 \pi} \frac{{ }^{\star} \rho_{L}(k x, k, T)}{x} \simeq \frac{2}{5 m^{2}} .
$$

All of the proposed solutions rely on one or another use of the Resummation Program, that is a one-loop resummation of leading order thermal fluctuations. In Refs. [13,14], for example, a resummation of multi-loop exchanges is performed à la Bloch-Nordsieck, within the one-loop effective theory provided by the Resummation Program. This approach, adequate in view of the ordering relation (3), yields a finite, regular damping rate, both in QED and QCD. Calculations, though, clearly differentiate the abelian QED, from the non-abelian case of QCD, whereas both situations are formally identical at this order. At Hard Thermal Loop order in effect, the QCD effective Lagrangian density 'abelianises' in that it only differs from the QED effective lagrangian density, by the relevant Lie-algebra valuation of the gauge fields, $A_{\mu}(x)$ in QED versus $\sum_{a} A_{\mu}^{a}(x) T^{a}$ in QCD, generators $T^{a}$ being taken in the appropriate representation [15].

The current article aims at taking advantage of this long standing issue in order to point out a structural peculiarity of perturbative treatments when applied to the high temperature quantum field theory context. This is done by means of an up to four-loop analysis of the fast muon damping rate, where the number of loops refers not to the effective perturbation theory, i.e., to the $R P$, but to bare perturbation theory and thus to the number of loops appearing in the basic diagram of Figure 1.

\section{Damping Rate at 3 Loop Order}

Loop orders are hereafter referred to bare real-time perturbation theory endowed with Advanced and Retarded free field propagators. For example, four-loop order refers to that of diagram of Figure 1, as the photon polarization tensor $\Pi_{R R}^{\mu v}(K)$ is itself evaluated at three-loop order, as discussed in Section 5 . 
Within the Resummation Program, one has

$$
\begin{aligned}
& { }^{\star} \rho_{T R / A}\left(k_{0}, k, T\right)=2 \operatorname{Im} \lim _{\eta \rightarrow 0^{+}} \frac{1}{K^{2}-\Pi_{T}\left(k_{0} \pm i \eta, k, T\right)}, \\
& { }^{\star} \rho_{L_{R / A}}\left(k_{0}, k, T\right)=2 \operatorname{Im} \lim _{\eta \rightarrow 0^{+}} \frac{K^{2}}{k^{2}} \frac{1}{K^{2}-\Pi_{L}\left(k_{0} \pm i \eta, k, T\right)},
\end{aligned}
$$

with the one-loop expressions of

$$
\Pi_{L} \rightarrow \Pi_{L}^{(1)}=-2 m^{2} \frac{K^{2}}{k^{2}}+m^{2} \frac{K^{2}}{k^{2}} \frac{k_{0}}{k} \ln \left(\frac{k_{0}+k}{k_{0}-k}\right), \text { and } \Pi_{T}^{(1)}=m^{2}-\Pi_{L}^{(1)} / 2
$$

and the thermal masses are [16],

$$
m_{\mathrm{QED}}^{2}=\frac{e^{2} T^{2}}{6}, \quad m_{\mathrm{QCD}}^{2}=\frac{g^{2} T^{2}}{9}\left(N_{c}+N_{f} / 2\right),
$$

for QCD with $N_{c}$ colors and $N_{f}$ flavors of quark fields.

As displayed in Equation (6), transverse and longitudinal degrees of freedom add up separately to the total damping rate (6), and the latter will be considered now. One has [5],

$$
\Pi_{L}^{(2)}\left(k_{0}, \vec{k}, T\right)=\frac{K^{2}}{k_{0} k_{z}}\left[\left(\Pi^{(S E)}\right)_{0 z}\left(k_{0}, \vec{k}, T\right)+\left(\Pi^{(V E)}\right)_{0 z}\left(k_{0}, \vec{k}, T\right)\right] \equiv \Pi_{L}^{(S E)}\left(k_{0}, \vec{k}, T\right)+\Pi_{L}^{(V E)}\left(k_{0}, \vec{k}, T\right),
$$

where $\Pi^{(S E)}$ and $\Pi^{(V E)}$ correspond to the two-loop pieces of Figures 2 and 3, respectively (Retarded/ Advanced indices, $R / A$, omitted for short).

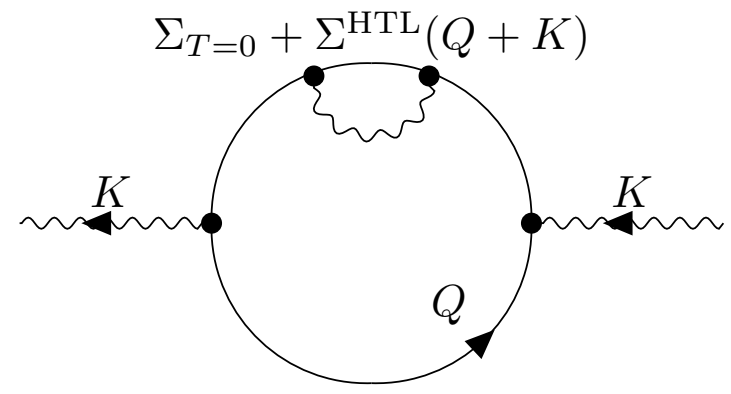

Figure 2. A two-loop contribution to the polarization tensor $\Pi^{(2)}\left(k_{0}, k\right)$.

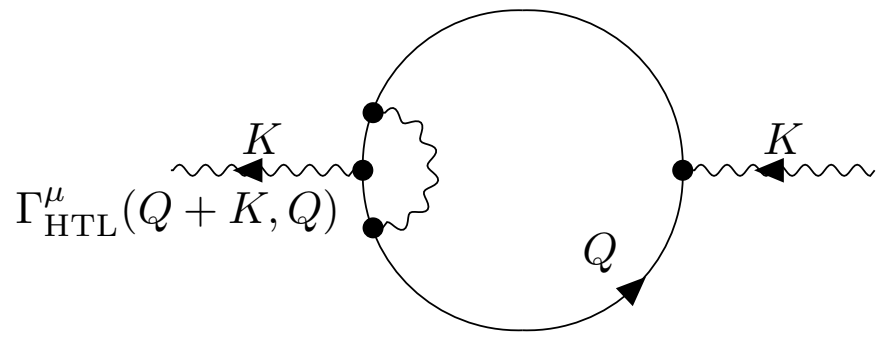

Figure 3. Another two-loop contribution to the polarization tensor $\Pi^{(2)}\left(k_{0}, k\right)$.

One finds (with $\varepsilon(x)=\Theta(x)-\Theta(-x)=\operatorname{sign}(x)$ ),

$$
\begin{gathered}
\Pi_{L}{ }^{(S E)}\left(k_{0}, \vec{k}, T\right)=-8 e^{2} m^{2} \frac{K^{2}}{k_{0} k_{z}} \int \frac{\mathrm{d}^{4} Q}{(2 \pi)^{3}}\left(1-2 n^{F}\left(q_{0}, T\right)\right) \varepsilon\left(q_{0}\right) \frac{\delta^{\prime}\left(Q^{2}\right)}{Q_{R}^{\prime 2}}\left(2 q_{0} q_{z}+q_{0} k_{z}+q_{z} k_{0}\right) \\
+\frac{4 i e^{2} K^{2}}{k_{0} k_{z}} \int \frac{\mathrm{d}^{4} Q}{(2 \pi)^{4}}\left(1-2 n^{F}\left(q_{0}\right)\right) \frac{1}{Q_{R}^{\prime 2}} \operatorname{disc}_{q_{0}} \frac{1}{Q_{R}{ }^{2}}\left(-q_{0}^{\prime} \Sigma_{z}-q_{z}^{\prime} \Sigma_{0}\right),
\end{gathered}
$$


and

$$
\begin{aligned}
\Pi_{L}(V E) & \left(k_{0}, \vec{k}, T\right)=+\frac{4 i e^{2} K^{2}}{k_{0} k_{z}} \int \frac{d^{4} Q}{(2 \pi)^{4}}\left(1-2 n^{F}\left(q_{0}, T\right)\right) \frac{1}{Q_{R}^{\prime 2}} \\
& \times \operatorname{disc}_{q_{0}} \frac{1}{Q_{R}{ }^{2}}\left[-m^{2} Q \cdot Q^{\prime} \int \frac{d \widehat{L}}{4 \pi} \frac{\widehat{L}_{0} \widehat{L}_{z}}{\left(\bar{L}_{Q}+i \epsilon\right)\left(\widehat{L}^{\prime}+i \epsilon\right)}+q_{z}^{\prime} \Sigma_{0}^{\prime}+q_{z} \Sigma_{0}\right],
\end{aligned}
$$

where $\widehat{L}=(1, \widehat{l})$ is a light-like four-vector, and where as in Equation (5), the usual Fermi-Dirac distribution $n_{F}(x)$ is deprived of any absolute value prescription. In (15) and (16), the mass squared term $m^{2}$ stands for either of the two QED and QCD possibilities of (13), and the shorthand notation of $\Sigma_{\mu} \equiv \Sigma_{\mu}(Q), \Sigma_{\mu}^{\prime} \equiv \Sigma_{\mu}\left(Q^{\prime}\right)$ is used, as well as $Q_{R}^{2}$ for $\left(q_{0}+i \eta\right)^{2}-\vec{q}^{2}$. The HTL-fermionic self-energy components are

$$
\Sigma_{R}^{\mu}(Q) \equiv m^{2} \int \frac{d \widehat{L}}{4 \pi} \frac{\widehat{L}^{\mu}}{\widehat{L} \cdot Q+i \epsilon},
$$

with the results,

$$
\Sigma_{\alpha}^{0}(Q)=\frac{m^{2}}{q} Q_{0}\left(\frac{q_{0}}{q}\right), \quad \Sigma_{\alpha}^{i}(Q)=\left(\frac{\vec{q}^{i}}{q} \equiv \widehat{q}^{i}\right) \frac{m^{2}}{q} Q_{1}\left(\frac{q_{0}}{q}\right) \quad \alpha=R, A,
$$

$Q_{0}$ and $Q_{1}$ being the Legendre functions,

$$
Q_{1}(x)=x Q_{0}(x)-1, \quad Q_{0}(x)=\frac{1}{2} \ln \frac{x+1}{x-1} .
$$

In this way, the $R / A$ prescriptions of Equation (19) are encoded in the logarithmic determinations of the Legendre functions. For completeness, the HTL vertex reads as

$$
\Gamma_{\mu}^{H T L}\left(Q, Q^{\prime}\right)=m^{2} \int \frac{d \widehat{L}}{4 \pi} \frac{\widehat{L}_{\mu} \widehat{L}}{(\widehat{L} \cdot Q+i \epsilon)\left(\widehat{L} \cdot Q^{\prime}+i \epsilon\right)} .
$$

It is convenient to choose the $z$-axis along the vector $\vec{k}$, so that $k_{z}=k$ and $q_{z}=q x$, with $x=(\widehat{k} \cdot \widehat{q})$. Out of Equations (16) and (17), several integrals come about,

$$
\begin{gathered}
I_{1}=-8 e^{2} m^{2} \frac{K^{2}}{k_{0} k} \int \frac{\mathrm{d}^{4} Q}{(2 \pi)^{3}}\left(1-2 n^{F}\left(q_{0}, T\right)\right) \varepsilon\left(q_{0}\right) \frac{\delta^{\prime}\left(Q^{2}\right)}{Q_{R}^{\prime 2}}\left(2 q_{0} q x\right), \\
I_{2}=-8 e^{2} m^{2} \frac{K^{2}}{k_{0}} \int \frac{\mathrm{d}^{4} Q}{(2 \pi)^{3}}\left(1-2 n^{F}\left(q_{0}, T\right)\right) \varepsilon\left(q_{0}\right) \frac{\delta^{\prime}\left(Q^{2}\right)}{Q_{R}^{\prime 2}}\left(q_{0}\right), \\
I_{3}=-8 e^{2} m^{2} \frac{K^{2}}{k} \int \frac{\mathrm{d}^{4} Q}{(2 \pi)^{3}}\left(1-2 n^{F}\left(q_{0}, T\right)\right) \varepsilon\left(q_{0}\right) \frac{\delta^{\prime}\left(Q^{2}\right)}{Q_{R}^{\prime 2}}(q x), \\
I_{4}=4 i e^{2} \frac{K^{2}}{k_{0} k} \int \frac{\mathrm{d}^{4} Q}{(2 \pi)^{4}}\left(1-2 n^{F}\left(q_{0}, T\right)\right) \frac{q_{z}^{\prime} \Sigma_{0}^{\prime}}{Q_{R}^{\prime 2}} \operatorname{disc} \frac{1}{Q_{R}^{2}}, \\
I_{5}=-4 i e^{2} \frac{K^{2}}{k_{0} k} \int \frac{\mathrm{d}^{4} Q}{(2 \pi)^{4}}\left(1-2 n^{F}\left(q_{0}, T\right)\right) \frac{q_{0}^{\prime}}{Q_{R}^{\prime 2}} \operatorname{disc} \frac{\Sigma_{z}}{Q_{R}^{2}}, \\
I_{6}=-4 i e^{2} \frac{K^{2}}{k_{0}} \int \frac{\mathrm{d}^{4} Q}{(2 \pi)^{4}}\left(1-2 n^{F}\left(q_{0}, T\right)\right) \frac{1}{Q_{R}^{\prime 2}} \operatorname{disc} \frac{\Sigma_{0}}{Q_{R}^{2}}, \\
I_{7}=-4 i e^{2} m^{2} \frac{K^{2}}{k_{0} k} \int \frac{\mathrm{d}^{4} Q}{(2 \pi)^{4}}\left(1-2 n^{F}\left(q_{0}, T\right)\right) \frac{Q \cdot Q^{\prime}}{Q_{R}^{\prime 2}} \operatorname{disc} \frac{1}{Q_{R}{ }^{2}} \int \frac{\mathrm{d} \widehat{L}}{4 \pi} \frac{\widehat{L}_{0} \widehat{L} z}{(\widehat{L} \cdot Q+i \epsilon)\left(\widehat{L} \cdot Q^{\prime}+i \epsilon\right)},
\end{gathered}
$$

and the leading order contributions to any of the seven above integrals will be retained in the limit of $k / T \ll 1$. Note that, at this stage, the definite order of magnitude of $k / T$ is not yet (and needs not 
being) determined. As it will turn out shortly, the specific order of magnitude of $k / T$ is selected by the two-loop order fluctuations themselves.

The first three integrals correspond to the HTL self energy insertion depicted in Figure 2 and yield

$$
\begin{aligned}
I_{1}+I_{2}+I_{3} & =-\frac{K^{2}}{k^{2}} \frac{e^{2} m^{2}}{\pi^{2}} Q_{1}\left(\frac{k_{0}}{k}\right)\left(\int_{0}^{\infty} \frac{\mathrm{d} x}{x} \frac{\mathrm{d}}{\mathrm{d} x} x \tanh \frac{x}{2}\right)^{\text {ren. }} \\
& +\frac{K^{2}}{k^{2}} \frac{e^{2} m^{2}}{4 \pi^{2}}\left(2 \frac{k_{0}}{k}-\frac{k}{k_{0}}\right) \int_{0}^{\infty} \frac{\mathrm{d} x}{x} \frac{\mathrm{d}}{\mathrm{d} x} x \tanh \frac{x}{2} \ln \frac{K_{+}^{2}-x^{2}}{K_{-}^{2}-x^{2}} \\
& +\frac{K^{2}}{k^{2}} \frac{e^{2} m^{2}}{2 \pi^{2}} \frac{T}{k} \int_{0}^{\infty} \frac{\mathrm{d} x}{x} \frac{\mathrm{d}}{\mathrm{d} x} x^{2} \tanh \frac{x}{2} \ln \frac{K_{+}+x}{K_{-}+x} \frac{K_{-}-x}{K_{+}-x} \\
& +\left(\frac{K^{2}}{k^{2}}\right)^{2} \frac{e^{2} m^{2}}{8 \pi^{2}} \frac{k}{T} \int_{0}^{\infty} \frac{\mathrm{d} x}{x} \frac{\mathrm{d}}{\mathrm{d} x} \tanh \frac{x}{2} \ln \frac{K_{+}+x}{K_{-}+x} \frac{K_{-}-x}{K_{+}-x} .
\end{aligned}
$$

With $K_{ \pm}=\left(k_{0} \pm k\right) / 2 T$, and accordingly $K_{ \pm}\left(-k_{0}, k\right)=-K_{\mp}\left(k_{0}, k\right)$, the parity properties of the Retarded determination of $\Pi_{L}^{(2)}\left(k_{0}, k\right)$ are met as they should.

For $I_{5}$, one finds

$$
I_{5}=\frac{e^{2} m^{2}}{\pi^{2}} \frac{K^{2}}{k^{2}} \frac{T}{k} Q_{0}\left(\frac{k_{0}}{k}\right) \int_{0}^{\infty} \mathrm{d} x\left(1-2 n_{F}(x)\right) .
$$

An apparet leading behaviour shows up with the factor $T / k$, but the whole contribution is ruled out by the odd $k_{0}$-parity of the Legendre function $Q_{0}$.

For $I_{6}$, one finds

$$
\operatorname{Re} I_{6}=\frac{e^{2} m^{2}}{2 \pi^{2}} \frac{K^{2}}{k_{0} k} \int \mathrm{d} q \int_{-q}^{+q} \frac{\mathrm{d} q_{0}}{q_{0}^{2}-q^{2}}\left[\varphi(q)-\varphi\left(q_{0}\right)\right] .
$$

with

$$
\varphi(q)=\tanh \left(\frac{q_{0}}{2 T}\right) \ln \frac{k_{0} q_{0}+k q}{k_{0} q_{0}-k q}
$$

for the real part, and

$$
\operatorname{Im} I_{6}=-\frac{e^{2} m^{2}}{4 \pi} \frac{K^{2}}{k_{0} k} \int_{0}^{\infty} \frac{\mathrm{d} x}{x} \tanh \frac{x}{2} \ln \frac{K_{+}+x}{K_{-}+x} \frac{K_{-}-x}{K_{+}-x}
$$

for the imaginary part. Intermediate mass singularities develop at $q_{0}= \pm q$, and cancel each other out in the end as further examples will display below.

For $I_{4}$, contributions are found to cancel out, due to $x$ - and $k_{0}$-odd parities.

For $I_{7}$, the calculations of [8-10] can be used (in particular Equation (3.15) in [9]) to yield

$$
\operatorname{disc}_{q_{0}} \int \frac{\mathrm{d} \widehat{L}}{4 \pi} \frac{\widehat{L}_{0} \widehat{L}_{z}}{(\widehat{L} \cdot Q+i \epsilon)\left(\widehat{L} \cdot Q^{\prime}+i \epsilon\right)}=i \pi \frac{q_{0}}{q} \varepsilon\left(q_{0}\right) \frac{\Theta\left(-Q^{2}\right)}{K \cdot Q+i \varepsilon k_{0}} .
$$

On the basis of the leading order expansion,

$$
\frac{Q \cdot Q^{\prime}}{Q_{R}^{\prime 2}}=1-\frac{K \cdot Q^{\prime}}{Q_{R}^{2}}+\mathcal{O}\left(\left(\frac{K \cdot Q^{\prime}}{Q_{R}{ }^{2}}\right)^{2}\right) .
$$

it is immediate to check that the contributions attached to Equation (34) are cancelled by the odd parity character of the integration on $q_{0}$.

Then, following [10], to take the discontinuity of $Q_{R}^{-2}$, one has first to dispose of the result,

$$
\begin{aligned}
& \int \frac{d \widehat{L}}{4 \pi} \frac{\widehat{L}_{0} \widehat{L}_{z}}{(\widehat{L} Q+i \epsilon)\left(\widehat{L} Q^{\prime}+i \epsilon\right)}=-\frac{1}{2 q q^{\prime}} \ln \left(\frac{q_{0}^{\prime}+q^{\prime}}{q_{0}^{\prime}-q^{\prime}}\right) \\
& -\frac{1}{2 \sqrt{(K \cdot Q)^{2}-K^{2} Q^{2}}} \ln \left(\frac{Q \cdot Q^{\prime}+\sqrt{(K \cdot Q)^{2}-K^{2} Q^{2}}}{Q \cdot Q^{\prime}-\sqrt{(K \cdot Q)^{2}-K^{2} Q^{2}}}\right),
\end{aligned}
$$


so as to proceed with the evaluation of $I_{7}$,

$$
\begin{gathered}
+4 i e^{2} m^{2} \frac{K^{2}}{k_{0} k} \int \frac{q \mathrm{~d} q}{(2 \pi)^{3}} \int_{-1}^{+1} \mathrm{~d} x \int \mathrm{d} q_{0} \tanh \left(\frac{q_{0}}{2 T}\right)(-2 i \pi) \varepsilon\left(q_{0}\right)\left(\delta\left(q_{0}-q\right)+\delta\left(q_{0}+q\right)\right) \\
\times\left\{\frac{1}{2 q q^{\prime}} \ln \left(\frac{q_{0}^{\prime}+q^{\prime}}{q_{0}^{\prime}-q^{\prime}}\right)+\frac{1}{2 K \cdot Q} \ln \frac{(2 Q \cdot K)^{2}}{K^{2} Q^{2}}\right\} .
\end{gathered}
$$

Considering the second term of the second line in curly brackets of (36), the sum on $q_{0}$ yields

$$
-\tanh \left(\frac{q}{2 T}\right) \frac{1}{2 q\left(k_{0}-k x\right)} \ln \frac{k_{0}-k x}{-\delta}
$$

where $\delta \rightarrow 0$ stands for $\left|q_{0}-q\right|$ when letting $q_{0} \rightarrow 0$. At $q_{0}=-q$, one obtains

$$
\tanh \left(\frac{q}{2 T}\right) \frac{1}{2 q\left(k_{0}+k x\right)} \ln \frac{-k_{0}-k x}{\delta} .
$$

Integrating on $x$, the mass singularities, at $\delta=0$, are, therefore,

$$
\ln (-\delta) \tanh \left(\frac{q}{2 T}\right) \frac{1}{2 q k} \ln \frac{k_{0}+k}{k_{0}-k}
$$

plus

$$
-\ln (\delta) \tanh \left(\frac{q}{2 T}\right) \frac{1}{2 q k} \ln \frac{k_{0}+k}{k_{0}-k}
$$

The sum of the two previous expressions cancels out the $\ln (\delta)$ singular terms which are plaguing the real part of (34), while the imaginary part is finite and reads

$$
\pm i \pi \tanh \left(\frac{q}{2 T}\right) \frac{1}{2 q k} \ln \frac{k_{0}+k}{k_{0}-k} .
$$

With the overall multiplicative factor of $K^{2} / k_{0} k$, this produces an irrelevant imaginary part (even in $k_{0}$ ).

The regular (real) contributions that are left read

$$
\frac{1}{2 q} \tanh \left(\frac{q}{2 T}\right)\left\{-\int_{-1}^{+1} \frac{\mathrm{d} x}{k_{0}-k x} \ln \frac{k_{0}-k x}{q}+\int_{-1}^{+1} \frac{\mathrm{d} x}{k_{0}+k x} \ln \frac{-k_{0}-k x}{q}\right\},
$$

and add up to a null result,

$$
\tanh \left(\frac{q}{2 T}\right) \frac{1}{2 q k}\left\{\ln \frac{k_{0}-k}{k_{0}+k} \ln \frac{K^{2}}{q^{2}}+\ln \frac{k_{0}+k}{k_{0}-k} \ln \frac{K^{2}}{q^{2}}\right\}=0 .
$$

For the first term in the second line of (36), one has at leading order $k / q \ll 1$, after having integrated over $q_{0}$,

$$
\frac{i \pi}{q^{2}} \tanh \left(\frac{q}{2 T}\right) \int_{-1}^{+1} \mathrm{~d} x \ln \left(\frac{2 q+k_{0}+k x}{k_{0}-k x} \frac{k_{0}+k x}{k_{0}-k x-2 q}\right) .
$$

Integration on $x$ gives no real part,

$$
\frac{k_{0}}{k} \ln \frac{k_{0}-k}{k_{0}+k}-\ln \frac{K^{2}}{4 q^{2}}+\frac{k_{0}}{k} \ln \frac{k_{0}+k}{k_{0}-k}+\ln \frac{K^{2}}{4 q^{2}}=0
$$


and a non-zero imaginary one,

$$
\pm i \frac{e^{2} m^{2}}{2 \pi} \frac{K^{2}}{k^{2}} \frac{k}{k_{0}}\left(\int_{0}^{\infty} \frac{\mathrm{d} x}{x} \tanh \frac{x}{2}\right)^{\text {ren. }}
$$

On the whole, at leading order, the evaluation of (36) yields no contribution to $I_{7}$ other than Equation (47) whose imaginary character comes from the only condition $k_{\mu} / q_{\mu} \ll 1$ and therefore is kinematically compatible with the overall constraint of Equation (6), which is $-k \leq k_{0} \leq k$.

Comments are in order. In Equation (27), the fourth contribution is by a factor $k / T$ - subleading and can be ignored. In the third line, a contribution of order $\left(K^{2} / k^{2}\right)\left(e^{2} m^{2} / 2 \pi^{2}\right)(T / k)$ seems to dominate all the other contributions by a factor of $T / k$. One has in effect

$$
e^{2} m^{2} \frac{K^{2}}{k^{2}}\left(\frac{T}{k}\right) C\left(k_{0}, k\right)=\frac{K^{2}}{6}\left(\frac{e^{2} T}{k}\right)^{2} \frac{T}{k} C\left(k_{0} / T, k / T\right),
$$

where the QED value of $m^{2}=e^{2} T^{2} / 6$ has been used for the sake of illustration, and where $C\left(k_{0} / T, k / T\right)$ denotes the function

$$
\begin{aligned}
& C\left(k_{0} / T, k / T\right)=\int_{0}^{\infty} \mathrm{d} x \tanh \left(\frac{x}{2}\right) \ln \frac{K^{2} / T^{2}-4 x(x+k / T)}{K^{2} / T^{2}-4 x(x-k / T)} \\
& =\int_{0}^{\infty} \mathrm{d} x \tanh \left(\frac{x}{2}\right) \ln \frac{[x+K+]\left[x-K_{-}\right]}{\left[x-K_{+}\right]\left[x+K_{-}\right]}
\end{aligned}
$$

with $K_{ \pm}$such as defined after (28). The function $C\left(k_{0} / T, k / T\right)$ is infrared regular, but ultraviolet singular. At large $x(=q / T)$, it exhibits the ultraviolet logarithmic divergence. It arises from the $T=0$-part of the self-energy diagram of Figure 2 and due to the factor 1 in $1-2 n_{F}(x)=\tanh (x / 2)$. One has, indeed,

$$
C\left(k_{0} / T, k / T\right) \sim \frac{k}{T} \int_{0}^{\infty} \frac{\mathrm{d} x}{x} \Longrightarrow \Pi^{(2)} \sim \frac{K^{2}}{6} \frac{e^{4} T^{2}}{k^{2}} \int_{0}^{\infty} \frac{\mathrm{d} x}{x} .
$$

One-loop renormalized at $T=0$, this ultraviolet logarithmic divergence is thus replaced by a (renormalization scheme dependent) constant $C^{s t}$, and the corresponding contribution to $\Pi_{L}^{(2)}$ therefore is $C^{s t}\left(K^{2} / 6\right)\left(e^{4} T^{2} / k^{2}\right)$. This also is the case of the non-zero $T$-part of $C\left(k_{0} / T, k / T\right)$. One finds [17],

$$
\begin{aligned}
& C_{T}\left(k_{0} / T, k / T\right) \equiv-2 \int_{0}^{\infty} \mathrm{d} x n_{F}(x), \ln \frac{K^{2} / T^{2}-4 x(x+k / T)}{K^{2} / T^{2}-4 x(x-k / T)} \simeq-e^{k / T} E_{i}(-k / T) \\
& +e^{-k / T}(\gamma+\ln k / T)-i \pi\left(1-e^{-k / T}\right)+e^{-k / T} \int_{0}^{1} \mathrm{~d} x \frac{e^{k x / T}-1}{x}
\end{aligned}
$$

where the approximate equality refers to the substitution of $e^{-x}$ for $n_{F}(x)$ and to the neglect of $K^{2} / T^{2}$ in the logarithm, and where $E_{i}$ is the exponential integral function. Note that this is consistent in view of Equation (6), which indicates that $k_{0}$ is on the same order of magnitude as $k$, and thus $K^{2} / T^{2} \ll k / T$. By preserving that term, however, the same result is reached.

Now, since $E_{i}(-k / T)=\gamma+\ln k / T+\mathcal{O}(k / T)$, with $\gamma$ the Euler's constant, the two first terms of Equation (51) yield $-2[\gamma+\ln (k / T)] \sinh (k / T)$ and are of order $k / T$, as are also the two remaining terms in the second line of Equation (51). As displayed in Figure 4, a numerical evaluation of the full, non-approximate $C_{T}\left(k_{0}, k\right)$ function of (48) confirms this conclusion, which can be summarised as follows:

$$
\mathcal{O}\left(\frac{T}{k} C_{T}\left(k_{0} / T, k / T\right)\right)=\mathcal{O}\left(\frac{T}{k} C^{\text {ren. }}\left(k_{0} / T, k / T\right)\right)=\mathcal{O}(1)
$$



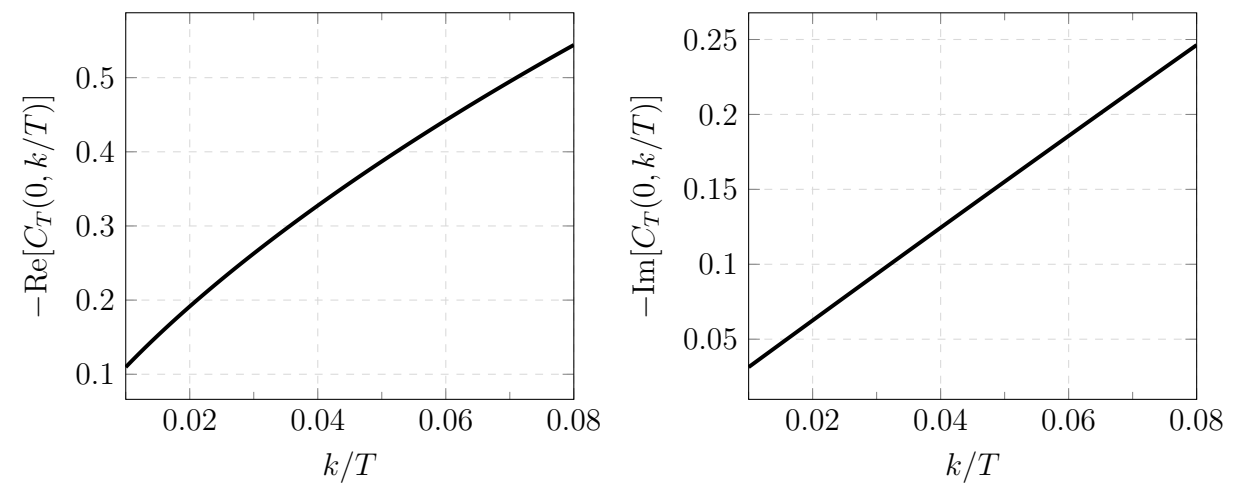

Figure 4. A plot of the (negatives of the) real and imaginary parts of Equation (51) as functions of $k / T$ for $K^{2} / T^{2}=0$.

To summarise, at two-loop order of bare perturbation theory and in the small $k$ / $T$-limit, inspection of expressions (22)-(28) reveals that there are no leading order contribution to $\Pi_{L}^{(2)}$ other than those of form $K^{2}\left(e^{4} T^{2} / k^{2}\right) \delta \mathcal{F}_{L}^{(2 ; \text { ren. })}$, where $\delta \mathcal{F}_{L}^{(2 ; \text {;ren.) }}$ denotes some dimensionless function of $k_{0} / T$ and $k / T$. In replacing $m^{2}$ of QED by the one of QCD, the same conclusion applies. Two remarks are in order:

(i) The notation used above, $\delta \mathcal{F}_{L}^{(2 ; \text { ren. })}$, instead of a would-be more natural notation like $F_{L}^{(2 ; r e n)}$ for example, is to mean that as well known $[18,19]$, not all of the leading order terms in the limit of $k / T \ll 1$ are accounted for by $\delta \mathcal{F}_{L}^{(2 ; r e n .)}$. This is explained below, after Equation (58).

(ii) For reasons that are made explicit in Section 6, Equation (104), in the present as well as in the following section, the contributions of transverse modes are not considered.

\section{Perturbative Peculiarity of the Thermal Case}

The diagrams of Figures 2 and 3 involve the gauge invariant HTL self energy (16) and vertex (17). By changing the gauge, only the (external) K-line propagator is changed, which doesn't impact the damping rate $\gamma(E, p, T)$ [20]. One is therefore tempted to conclude that by construction these two contributions to the photonic polarisation tensor, $\Pi_{\mu \nu}^{(2 ; S E)}$ and $\Pi_{\mu \nu}^{(2 ; V E)}$, are separately gauge invariant. This, however, needs to be checked. Calculations are long but straightforward and one finds effectively,

$$
K^{\mu} \Pi_{\mu \nu}^{(2 ; S E)}=0, \quad K^{\mu} \Pi_{\mu \nu}^{(2 ; V E)}=0 .
$$

Moreover. these relations hold at leading $k / T$-order, exemplifying that the principle of local gauge invariance is so deeply rooted in the theories that it is verified at any consistently determined momentum scale.

Taking advantage of the last results, Equation (12) could now be improved by writing

$$
{ }^{(2)} \rho_{L}\left(k_{0}, k, T\right)=2 \operatorname{Im} \frac{K^{2}}{k^{2}} \frac{1}{K^{2}-\Pi_{L}^{(1)}\left(k_{0}, k, T\right)-\Pi_{L}^{(2 ; \text { ren. })}\left(k_{0}, k, T\right)+i \varepsilon},
$$

and used to calculate the longitudinal contribution to the moving fermion damping rate at this order of approximation. According to the previous section, one has (QED case chosen for the sake of illustration)

$$
\Pi_{L}^{(2 ; \text { ren. })}\left(k_{0}, k, T\right)=K^{2}\left[\frac{e^{2} T}{k}\right]^{2} \delta \mathcal{F}_{L}^{(2 ; \text { ren. })}\left(k_{0}, k, T\right),
$$

where $\delta \mathcal{F}_{L}^{(2 ; \text { ren. })}\left(k_{0}, k, T\right)$ collects the various leading order contributions of (22)-(28) in the small $k / T$-limit, into some complex-valued function of $k_{0}$ and $k$. Like the Legendre function $Q_{1}\left(k_{0} / k\right)$ of the one-loop order, (13) and (19), $\delta \mathcal{F}_{L}^{(2 ; r e n .)}\left(k_{0}, k, T\right)$ is assumed to preserve the $K^{2}\left(e^{4} T^{2} / k^{2}\right)$ - leading 
order of magnitude of two-loop contributions to $\Pi_{L}^{(2)}\left(k_{0}, k\right)$. Still, the rather academic content of this assumption will be discussed in Section 7. Re-writing Equation (54),

$$
\rho_{L}^{(2)}\left(k_{0}, k, T\right)=2 \operatorname{Im} \frac{K^{2}}{k^{2}} \frac{1}{K^{2}\left(1+2 \frac{m^{2}}{k^{2}}\left[Q_{1}\left(k_{0} / k\right)+\frac{e^{2}}{2} \delta \mathcal{F}_{L}^{(2 ; \text { ren. })}\left(k_{0}, k, T\right)\right]+i \varepsilon\right)},
$$

it is made clear that, like in the $T=0$ usual perturbative context, the two-loop contribution $\Pi_{L}^{(2 ; \text { ren. })}\left(k_{0}, k, T\right)$ represents but an order $e^{2}$-correction to $\Pi_{L}^{(1)}\left(k_{0}, k\right) \equiv \Pi^{H T L}\left(k_{0}, k, T\right)$, the Hard Thermal Loop counterpart, and doesn't bring the fermion damping rate anything else than a correction on the same $e^{2}$-order of magnitude, that is, using Equation (56) instead of ${ }^{\star} \rho_{L}\left(k_{0}, k\right)$, one would get basically,

$$
\gamma(E, \vec{p}, T)=\frac{e^{2} T}{2 \pi}\left[C^{s t}+\mathcal{O}\left(e^{2}\right)\right]
$$

On the other hand, considering $\Pi_{L}^{(2 ; \text { ren. })}\left(k_{0}, k\right)$ in Equation (55), it is clear that, over momenta of order of $e^{2} T$, one has $\mathcal{O}\left(\Pi_{L}^{(2 ; \text { ren. })}\left(k_{0}, k\right)\right)=\mathcal{O}\left(K^{2}\right)$, so that any number of $\Pi_{L}^{(2 ; \text { ren. })}\left(k_{0}, k\right)$-insertions along the $K$-line will contribute a similar amount to the longitudinal degree of the internal $K$-line and must be resummed. For momenta of order $e^{2} T$ exclusively, this separated resummation leads to a spectral density of form,

$$
{ }^{(2)} \rho_{L}\left(k_{0}, k, T\right)=2 \operatorname{Im} \frac{K^{2}}{k^{2}} \frac{1}{K^{2}-\Pi_{L}^{(2 ; \text { ren. })}\left(k_{0}, k, T\right)+i \varepsilon},
$$

and, as displayed in next Section 5, the matter with Equation (58), and further with Equation (84), is that it contributes the fermionic damping rate $\gamma(E, p)$ on the same order of magnitude as the Hard Thermal Loop spectral density itself, ${ }^{\star} \rho_{L}\left(k_{0}, k, T\right) \equiv{ }^{(1)} \rho_{L}\left(k_{0}, k, T\right)$, that is on the leading order of $e^{2} T$.

This situation has no equivalent in the perturbative context of $T=0$ theories and appears to be peculiar to the non-zero $T$-case. In QCD, the necessity of resumming 'ultra-soft' order $g^{2} T$-fluctuations into another effective perturbation theory was recognized years ago on the basis of an equivalent semi-classical approach [19] to high- $T$ quantum field theories, and the new effective theory was shown to enjoy the same symmetry properties as those of the HTL-resummation program:

(1) For external momenta $k_{0} \leq k \leq g^{2} T$, ultra-soft amplitudes are as large as the corresponding HTL and tree-level ones, (2) they are gauge fixing independent (covariant and Coulomb-like gauges ...), and (3) ultrasoft amplitudes satisfy simple Ward identities. (4) Unlike the soft amplitudes controlled by the resummation program, though, ultra-soft amplitudes receive contributions from an infinite series of multi-loop diagrams ('ladder diagrams', to be specific).

It is therefore natural to speculate that a higher number of loop contributions could generate similar conclusions, with gauge invariant fluctuations at smaller momentum scales (orders $e^{3} T, e^{4} T$, etc.), generating for the cut photonic propagator $D_{\mu v}^{+}(K)$ of Equation (4) contributions all on the same order of magnitude as the tree-level ones.

The entire perturbative structure would therefore have to be considered along with layers of momentum scales, each of them controlled by a specific effective theory. Point (4), however, seems to cast some doubts on this expectation. In effect, the ultra-soft effective perturbation theory is taken to be terminal, as the ultra-soft denomination itself is intended to mean. In the next section, though, it is ventured that this could not be true, due to a specific interplay of $T=0$-renormalized with $T \neq 0$ Hard Thermal Loop vertices.

Though contributing equally to the net fermion damping rate (see Equation (6)), transverse contributions have not been considered for reasons to be given explicitly in Section 6 . 


\section{Damping Rate at Four-Loop Order}

In this section, three-loop contributions to $D_{\mu \nu}^{+}(K)$ are considered such as pictured in Figure 5, contributing $\gamma(E, p, T)$ at four-loop order. One can start with two self energy insertions $\Sigma_{R}(Q)$ and $\Sigma_{R}\left(Q^{\prime}\right)$, which at one-loop order and in real-time formalisms come about additive in their $T=0$ and $T \neq 0$ parts, $\Sigma_{T=0}$ and $\Sigma^{H T L}$, Equations (18) and (19). Various terms contribute to $\Pi_{L}^{(3 ; \text { ren.) }}\left(k_{0}, k\right)$, whose leading terms in the small $k_{\mu} / T$-limit admit the generic form,

$$
\delta \Pi_{L}^{(3 ; \text { ren. })}\left(k_{0}, k, T\right) \sim K^{2}\left[\frac{e^{2} T}{k}\right]^{3} \delta F_{L}^{(3 ; \text { ren. })}\left(k_{0}, k, T\right),
$$

with some dimensionless associated functions $\delta F_{L}^{(3 ; \text { ren. })}$, possibly one- and/or two-loop renormalized at $T=0$. Clearly, in line with the expectations of the semi-classical analyses [19], these contributions are to be considered as part of the ultra-soft amplitudes for $\mathcal{O}\left(k_{\mu}\right) \sim e^{2} T$ and do not define any new momentum scale of invariant fluctuations. Examples are as follows.

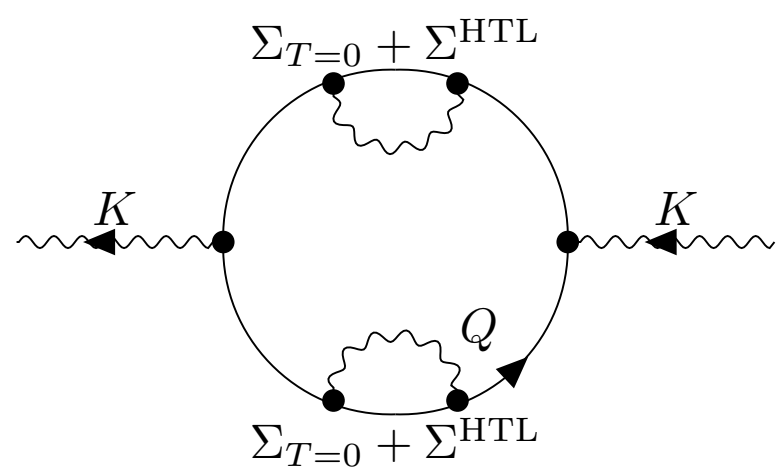

Figure 5. A three-loop contribution to the polarization tensor $\Pi^{(3)}\left(k_{0}, k, T\right)$.

As bare power counting suggests, one may replace one of the two self energy insertions by its $T=0$-renormalized part only, $\Sigma_{T=0}^{\text {ren. }}(Q)$. At $T=0$, though, the fermionic self energy is not gauge invariant. In the Coulomb gauge being chosen, one has [21],

$$
\Sigma^{\text {ren. }}(Q)=\frac{\alpha}{4 \pi} C^{\text {ren. }} Q+\frac{\alpha}{4 \pi}\left[\frac{19}{6} \vec{\gamma} \cdot \vec{q}-\frac{1}{2} \gamma^{0} q^{0}+\ldots\right],
$$

so that one could expect contributions to $\Pi_{L}^{(3 ; \text { ren. })}\left(k_{0}, k\right)$, on the order of $e^{2} \alpha m^{2}=\mathcal{O}\left(e^{6} T^{2}\right)$, to be a good candidate for a new relevant momentum scale at order $e^{3} T$. Now, besides a lack of gauge invariance, explicit calculations of the various terms stick to the behaviour (59) and hence, at best, would only be a part of the ultra-soft momentum scale fluctuations.

The same applies as well to two $T=0$ self energy insertions $\Sigma^{\text {ren. }}(Q)$ and $\Sigma^{\text {ren. }}\left(Q^{\prime}\right)$. For example, among other contributions, one has with the first term of Equation (60) (in the small $k_{\mu} / T \ll 1$ limit always),

$$
\begin{gathered}
\delta \Pi_{L}^{(3)}\left(k_{0}, k, T\right) \simeq-i \frac{K^{2}}{k_{0} k_{z}}(-i e)^{2}\left(\alpha C^{\text {ren. }}\right)^{2} \int \frac{q^{2} \mathrm{~d} q}{(2 \pi)^{3}} \int_{-1}^{+1} \mathrm{~d} x \int \mathrm{d} q_{0} \tanh \left(\frac{q_{0}}{2 T}\right) \\
\frac{1}{2 Q \cdot K} \frac{-i \pi}{q} \varepsilon\left(q_{0}\right)\left[\delta\left(q_{0}-q\right)+\delta\left(q_{0}+q\right)\right]\left[2 q_{0} q x+k_{0} q x+q_{0} k\right] .
\end{gathered}
$$

Explicit calculations give $\delta F_{L}^{(3 ; \text { ren. })}\left(k_{0}, k, T\right)=0$ for the leading term $2 q_{0} q x$ in the second line of Equation (61), while, for the second (sub-leading term) $k_{0} q x$, one finds

$$
\frac{K^{2}}{2}\left(\frac{C^{\text {ren. }}}{8 \pi}\right)^{2}\left[\frac{e^{2} T}{k}\right]^{3} \delta F_{L}^{(3 ; \text { ren. })}\left(k_{0}, k, T\right)
$$


with

$$
\delta F_{L}^{(3 ; \text { ren. })}\left(k_{0}, k, T\right)=\frac{k}{T}\left[\ln \left|\frac{k_{0}^{2}+k^{2}}{k_{0}^{2}-k^{2}}\right|-i \pi \Theta\left(-K^{2}\right)\right]\left(\int_{0}^{\infty} \mathrm{d} x x \tanh \frac{x}{2}\right)^{\text {ren. }},
$$

that is, besides an irrelevant $k_{0}$-even imaginary part, an order $k / T$ subleading real contribution. The last term in the second line of Equation (61), $q_{0} k$, yields another contribution corresponding to a new function,

$$
\delta F_{L}^{(3 ; \text { ren. })}\left(k_{0}, k, T\right)=\frac{k}{T}\left[\frac{k}{k_{0}} \ln \left|\frac{k_{0}+k}{k_{0}-k}\right|-i \pi \frac{k}{k_{0}} \Theta\left(-K^{2}\right)\right]\left(\int_{0}^{\infty} \mathrm{d} x x \tanh \frac{x}{2}\right)^{\text {ren. }},
$$

with correct parities but an order $k / T$ sub-leading as well.

All in all, inspection of the various terms shows that the contributions to $\Pi_{L}^{(3)}\left(k_{0}, k, T\right)$ due to the fluctuations depicted in Figure 5 can be written in the form (59) with related $\mathcal{O}(1)$ functions $\delta F_{L}^{(3 ; \text { ren. })}$ for the leading order ones. More precisely, within the topology of Figure 5, contributions of order $\left(e^{3} T / k\right)^{2}$ are effectively met, but, compared to $\left(e^{2} T / k\right)^{3}$-ones, they are sub-leading by a factor of $k / T$. Accordingly, the contributions attached to the topology of Figure 4 (some of them retaining further dependences on the Coulomb gauge, what is more) could at best complete the contributions attached to fluctuations at ultra-soft momentum scale, but can in no way define any new gauge invariant fluctuation of the internal photonic line at a softer momentum scale, such as $e^{3} T$.

It is with the diagram of Figure 6 that things start to appear to be different. This is because the zero temperature one-loop renormalized vertex reads $(\mathrm{QED})$

$$
\Gamma_{\mu}^{R}\left(Q^{\prime}, Q\right)=\gamma_{\mu} F_{1}\left(K^{2}\right)+\frac{i}{2 m} \sigma^{\mu v} k_{\nu} F_{2}\left(K^{2}\right),
$$

with $F_{i}\left(K^{2}\right), i=\{1,2\}$ representing the electric and magnetic form factors. Using this form of the vertex will be discussed below. The trace over Dirac indices, which are involved in this diagram, are

$$
F_{1}\left(K^{2}\right) \operatorname{Tr}\left[\gamma^{\mu} \frac{i Q}{Q_{R}^{2}} \frac{Z^{H T L}(Q)}{i} \frac{i Q}{Q_{R}^{2}} \gamma^{v} \frac{i Q^{\prime}}{Q^{\prime 2}}\right]
$$

as the form factor $F_{2}\left(K^{2}\right)$ will not contribute at this number of loops because of an odd number of Dirac matrices. Evaluating Equation (66) at $\mu=0, v=3$ in order to get $\Pi_{L}^{(3)}$, the result is

$$
\begin{aligned}
& \left(\frac{K^{2}}{k_{0} k}\right) \times-F_{1}\left(K^{2}\right)\left(\frac{1}{Q_{R}^{2}}\right)^{2} \frac{1}{Q^{\prime 2}}\left[8 m^{2}\left(2 q_{0} q x+k_{0} q x+q_{0} k\right)\right. \\
& \left.-4 Q^{2} \frac{m^{2}}{q}\left\{-x\left(q_{0}+k_{0}\right)+\left[k+x \frac{q^{2}+q_{0}^{2}+q_{0} k_{0}}{q}\right] Q_{0}\left(\frac{q_{0}}{q}\right)\right\}\right] .
\end{aligned}
$$

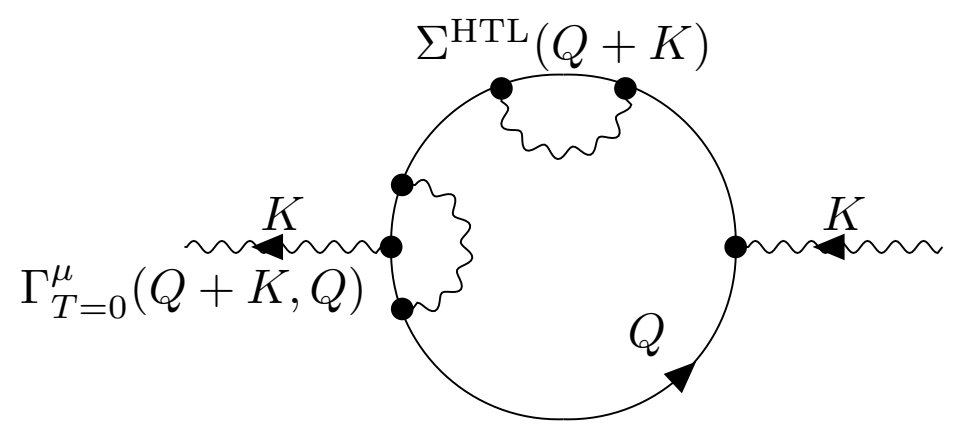

Figure 6. Another three-loop contribution to the polarization tensor $\Pi^{(3)}\left(k_{0}, k, T\right)$. 
Integrated over $Q$, Equation (67) exhibits mass singularities whose cancellations can be seen as follows.

The first line of Equation (67) is easily evaluated to give

$$
-\frac{1}{\pi} K^{2} \frac{e^{2} m^{2} F_{1}\left(K^{2}\right)}{k^{2}}\left[\frac{k_{0}}{k} Q_{0}\left(\frac{k_{0}}{k}\right)+Q_{1}\left(\frac{k_{o}}{k}\right)\right]\left(\int_{0}^{\infty} \frac{\mathrm{d} x}{x} \frac{\mathrm{d}}{\mathrm{d} x} x \tanh \frac{x}{2}\right)^{\text {ren. }} .
$$

Within the integration range of (5), a non-trivial imaginary part develops, which is essential to a non vanishing spectral density ${ }^{(3)} \rho_{L}\left(k_{0}, k, T\right)$,

$$
i \Theta\left(-K^{2}\right) K^{2} \frac{e^{2} m^{2} F_{1}\left(K^{2}\right)}{k^{2}}\left[\frac{k_{0}}{k}\right]\left(\int_{0}^{\infty} \frac{\mathrm{d} x}{x} \frac{\mathrm{d}}{\mathrm{d} x} x \tanh \frac{x}{2}\right)^{\text {ren. }} .
$$

This evaluation of the imaginary part is exactly what is obtained by putting the two fermionic lines on mass shell in agreement with the use of Equation (65) for the $T=0$ part of the one-loop renormalized vertex correction.

Turning to the second line of Equation (67), the first term in curly brackets (not proportional to $\left.Q_{0}\left(q_{0} / q\right)\right)$ contributes a term,

$$
K^{2} \frac{e^{2} m^{2} F_{1}\left(K^{2}\right)}{k^{2}} \frac{1}{4 \pi^{2}}\left[Q_{1}\left(\frac{k_{0}}{k}\right)\right]\left(\int_{0}^{\infty} \frac{\mathrm{d} x}{x} \tanh \frac{x}{2}\right)^{\text {ren. }} .
$$

with an associated imaginary part of

$$
-i \Theta\left(-K^{2}\right) K^{2} \frac{e^{2} m^{2} F_{1}\left(K^{2}\right)}{k^{2}} \frac{1}{8 \pi}\left[\frac{k_{0}}{k}\right]\left(\int_{0}^{\infty} \frac{\mathrm{d} x}{x} \tanh \frac{x}{2}\right)^{\text {ren. }} .
$$

For the second term in curly brackets in the second line of Equation (67) (proportional to $Q_{0}\left(k_{0} / k\right)$ ), two non-vanishing $q_{0}$-discontinuities can be taken.

Taking the discontinuity of $Q_{R}^{-2}$, the first one gets

$$
\begin{aligned}
& -K^{2} \frac{e^{2} m^{2} F_{1}\left(K^{2}\right)}{k^{2}} \frac{1}{8 \pi^{2}}\left[\frac{k}{k_{0}} Q_{0}\left(\frac{k_{0}}{k}\right)\right]\left[\int_{0}^{\infty} \frac{\mathrm{d} x}{x} \tanh \frac{x}{2}\right] \ln \left(\frac{2 q}{-\delta}\right), \\
& -K^{2} \frac{e^{2} m^{2} F_{1}\left(K^{2}\right)}{k^{2}} \frac{1}{8 \pi^{2}}\left[Q_{1}\left(\frac{k_{0}}{k}\right)\right]\left[\int_{0}^{\infty} \frac{\mathrm{d} x}{x} \tanh \frac{x}{2}\right] \ln \left(\frac{2 q}{-\delta}\right),
\end{aligned}
$$

where as before, $\delta=\left(q-\left|q_{0}\right|\right) \rightarrow 0$, regularises mass singularities at $q_{0}= \pm q$.

Then, taking the discontinuity of $Q_{0}\left(q_{0} / q\right)$ (i.e., $\left.-i \pi \Theta\left(-Q^{2}\right)\right)$, one obtains

$$
-\frac{K^{2}}{k_{0} k} \frac{e^{2} m^{2} F_{1}\left(K^{2}\right)}{16 \pi^{2}} \int \mathrm{d} q \int_{-q}^{+q} \frac{\mathrm{d} q_{0}}{2 q_{0}} \tanh \frac{q_{0}}{2 T}\left(\frac{1}{q_{0}-q}+\frac{1}{q_{0}+q}\right) \Phi\left(q_{0}, q ; k_{0}, k\right),
$$

with $\Phi\left(q_{0}, q ; k_{0} \cdot k\right)$ the function,

$$
\Phi\left(q_{0}, q ; k_{0}, k\right)=\ln \frac{k_{0} q_{0}+k q}{k_{0} q_{0}-k q}-2 \frac{q^{2}+q_{0}^{2}+q_{0} k_{0}}{q k}\left(1-\frac{k_{0} q_{0}}{q k} \ln \frac{k_{0} q_{0}+k q}{k_{0} q_{0}-k q}\right) .
$$

This function is a regular function of $q_{0}$ at $q_{0}= \pm q$, which allows for an isolation of singular behaviours in Equation (73). At $q_{0}=q-\delta$, one obtains

$$
-K^{2} \frac{e^{2} m^{2} F_{1}\left(K^{2}\right)}{16 \pi^{2} k^{2}}\left[\frac{k}{k_{0}} Q_{0}\left(\frac{k_{0}}{k}\right)\right]\left[\int_{0}^{\infty} \frac{\mathrm{d} x}{x} \tanh \frac{x}{2}\right]^{\text {ren. }} \ln \left(\frac{\delta}{-2 q}\right),
$$




$$
\begin{aligned}
& -K^{2} \frac{e^{2} m^{2} F_{1}\left(K^{2}\right)}{16 \pi^{2} k^{2}}\left[Q_{1}\left(\frac{k_{0}}{k}\right)\right]\left[\int_{0}^{\infty} \frac{\mathrm{d} x}{x} \tanh \frac{x}{2}\right]^{\text {ren. }} \ln \left(\frac{\delta}{-2 q}\right), \\
& -K^{2} \frac{e^{2} m^{2} F_{1}\left(K^{2}\right)}{8 \pi^{2} k^{2}}\left[Q_{1}\left(\frac{k_{0}}{k}\right)\right]\left[\int_{0}^{\infty} \frac{\mathrm{d} x}{x} \tanh \frac{x}{2}\right]^{\text {ren. }} \ln \left(\frac{\delta}{-2 q}\right) .
\end{aligned}
$$

On the other hand, at $q_{0}=-q+\delta$, one has

$$
\begin{aligned}
& +K^{2} \frac{e^{2} m^{2} F_{1}\left(K^{2}\right)}{16 \pi^{2} k^{2}}\left[\frac{k}{k_{0}} Q_{0}\left(\frac{k_{0}}{k}\right)\right]\left[\int_{0}^{\infty} \frac{\mathrm{d} x}{x} \tanh \frac{x}{2}\right]^{\text {ren. }} \ln \left(\frac{2 q}{\delta}\right), \\
& -K^{2} \frac{e^{2} m^{2} F_{1}\left(K^{2}\right)}{16 \pi^{2} k^{2}}\left[Q_{1}\left(\frac{k_{0}}{k}\right)\right]\left[\int_{0}^{\infty} \frac{\mathrm{d} x}{x} \tanh \frac{x}{2}\right]^{\text {ren. }} \ln \left(\frac{2 q}{\delta}\right) .
\end{aligned}
$$

Now, the first line of Equation (75) and the first line of Equation (76) compensate exactly the first line of Equation (72), while the second lines of Equations (75) and (76) cancel each other out. In the end, the second line of Equation (72) and the third line of Equation (75) cancel each other out exactly.

The regular contribution of the second group of terms in the second line line of Equation (67) thus is

$$
\begin{aligned}
& -K^{2} \frac{e^{2} m^{2} F_{1}\left(K^{2}\right)}{16 \pi^{2} k^{2}}\left[\frac{k}{k_{0}}\right] \int \mathrm{d} x \int_{-x}^{+x} \mathrm{~d} x_{0}\left(\frac{1}{x_{0}-x}+\frac{1}{x_{0}+x}\right) \\
& {\left[\frac{\tanh \left(x_{0} / 2\right)}{x_{0}} \Phi\left(T x_{0}, T x ; K\right)-\frac{\tanh (x / 2)}{x} \Phi(T x, T x ; K)\right],}
\end{aligned}
$$

and, due to $k_{0}$-improper parities, inducing cancellations in the integration over $k_{0}$, gets eventually reduced to

$$
-K^{2} \frac{e^{2} m^{2} F_{1}\left(K^{2}\right)}{8 \pi^{2} k^{2}}\left[\frac{k}{k_{0}} \int_{0}^{\infty} \mathrm{d} x \int_{0}^{x} \frac{\mathrm{d} x_{0}}{x_{0}-x}\left[\frac{\tanh \frac{x_{0}}{2}}{x_{0}} \ln \frac{k_{0} x_{0}+k x}{k_{0} x_{0}-k x}-\frac{\tanh \frac{x}{2}}{x} \ln \frac{k_{0}+k}{k_{0}-k}\right]\right] .
$$

For the regular part of $\delta \Pi_{L}^{(3 ; \text { ren.) }}$, however, only those terms for which the $Q$ and $Q^{\prime}$ internal lines can be considered on mass shell (i.e., $Q^{2}=0$ ), or approximately $\left(Q^{\prime 2} \simeq 0\right.$ ), should be retained so as to be consistent with the use of Equation (65) in lieu of the most general form of the e.m. vertex (as will be argued later, 3rd and 6th items below, this limitation does not hamper the point of the current paper).

All in all, the contributions (68) and (70) only are retained for $\delta \Pi_{L}^{(3 ; \text { ren.) }}\left(k_{0}, k, T\right)$ and stand for a part of the leading order contributions generated by the quantum fluctuation depicted in Figure 5 (remark (ii) below):

$$
\delta \Pi_{L}^{(3 ; \text { ren. })}\left(k_{0}, k, T\right)_{\text {leading }}=-K^{2}\left[\frac{e^{2} m^{2} F_{1}\left(K^{2}\right)}{k^{2}}\right] \delta \mathcal{F}_{L}^{(3 ; \text { ren. })}\left(k_{0}, k, T\right),
$$

with the function

$$
\delta \mathcal{F}_{L}^{(3 ; \text { ren. })}=\frac{1}{\pi}\left[\frac{k_{0}}{k} Q_{0}\left(\frac{k_{0}}{k}\right)+Q_{1}\left(\frac{k_{o}}{k}\right)\right] \times\left(\int_{0}^{\infty} \frac{\mathrm{d} x}{x} \frac{\mathrm{d}}{\mathrm{d} x} x \tanh \frac{x}{2}\right)^{\text {ren. }}-\frac{1}{4 \pi^{2}} Q_{1}\left(\frac{k_{o}}{k}\right)\left(\int_{0}^{\infty} \frac{\mathrm{d} x}{x} \tanh x\right)^{\text {ren. }} .
$$

At spacelike $K$-momenta, Equation (80) develops a non-zero imaginary part that is essential to a non vanishing spectral density ${ }^{(3)} \rho_{L}\left(k_{0}, k, T\right)$.

Due to the form factor $F_{1}\left(K^{2}\right)$, a non-trivial effect comes into play between the invariant $T=0$-renormalized vertex (65) and $H T L$ - self energy $\Sigma_{R}^{\mu}(Q)$. When renormalized, in effect, $F_{1}\left(K^{2}\right)$ is gauge-invariant to all orders in perturbation theory [22]. Since $\Sigma_{R}^{H T L}(Q)$ is also gauge-invariant, the fluctuation of Figure 5 brings to the cut photon propagator, $D_{\mu v}^{+}(K)$, a correction which is gauge-invariant by construction; or, more precisely, which doesn't retain more gauge dependence than the original one-loop HTL expression ${ }^{\star} D_{\mu \nu}^{+}(K)$ (as noticed before Equation (53), this remaining gauge-dependence is not problematic). This gauge invariance, by construction, can be checked also by explicit calculation and is here a mere consequence of Equation (53). 
So far, as displayed for example in the ordering relation (3), fermionic bare/renormalized masses have been ignored in front of the energy scales involved in the case considered. In the literature though, the one-loop form factor $F_{1}$ is always given at non zero fermionic masses and reads [22],

$$
F_{1}\left(K^{2}\right)=-\frac{\alpha}{\pi}\left[\left(1+\ln \frac{\mu}{m_{e}}\right)(1-\theta \operatorname{coth} \theta)+2 \operatorname{coth} \theta \int_{0}^{\theta / 2} \mathrm{~d} \varphi \varphi \tanh \varphi+\frac{\theta}{4} \tanh \frac{\theta}{2}\right],
$$

where $\sinh ^{2}(\theta / 2)=-K^{2} / 4 m_{e}^{2}$, and where $\mu^{2}$ is a small photon mass introduced so as to regularise intermediate infrared singular behaviours (mass singularities indeed). For reasons to be explained shortly, in line with the use of Equation (65), this logarithmic term can be consistently ignored. However, the $K^{2}$-dependence of $F_{1}$ remains fairly involved. A small $-K^{2} / 4 m_{e}^{2}$ limit of $F_{1}$ exists, and when such a regime becomes relevant to the high temperature context of either QED or QCD, Equation (81) simplifies to (QED case and ignoring the logarithmic term) [22],

$$
F_{1}\left(K^{2}\right) \simeq-\frac{\alpha}{8 \pi} \frac{K^{2}}{m_{e}^{2}}
$$

This form will be used to illustrate the main point of the current paper. Note that, in the case of QED, at $T=100 \mathrm{MeV}$ and $m_{e}=0.51 \mathrm{MeV}$, one obtains, at $\alpha=1 / 137$, a value of $\theta, 0 \leq \theta / 2 \leq$ arcsinh 0.12, which renders admissible the use of Equation (82) for $K$-momenta on the order of $e^{3} T$. The following remarks are in order:

(i) Using Equation (65) for the e.m. vertex amounts to reduce $\Pi_{\mu v}^{(3 ; \text { ren.) }}$ in Figure 6 to the contribution where the internal lines $Q$ and $Q^{\prime}$ are on mass shell, as illustrated in Figure 7, whereas the full imaginary part of $\Pi_{\mu \nu}^{(3 ; \text { ren.) }}$ entails also the cut diagram of Figure 8.

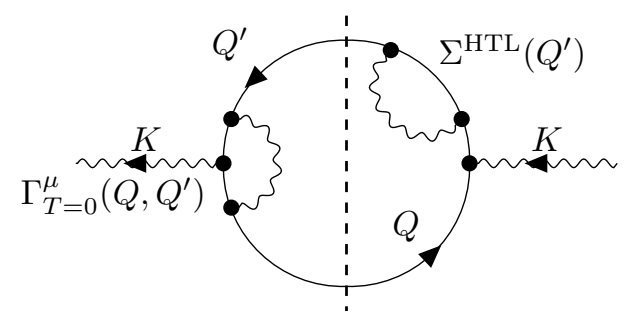

Figure 7. A contribution to the imaginary part of the polarization tensor $\Pi^{(3)}$. Cut lines $Q$ and $Q^{\prime}$ are on mass shell.

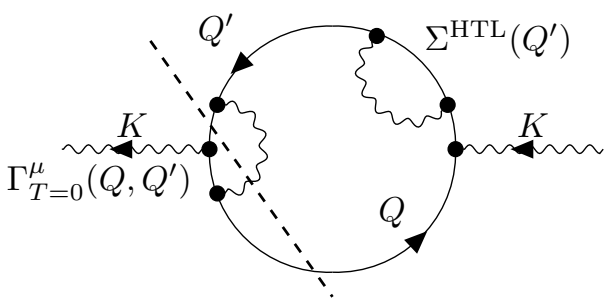

Figure 8. Another cut of the same diagram, contributing to the imaginary part of the polarization tensor $\Pi^{(3)}$.

Both contributions involve the mass singularity of Equation (81), that is, the logarithmic term $\ln \mu^{2} / m_{e}^{2}$, in such a way that these intermediate mass singularities cancel out in the sum, in agreement with standard $T=0$ situation (theorem $K L N$ [23]). As intermediate terms, which are absent of the final result for $\operatorname{Im} \Pi_{\mu v}^{(3 ; \text { ren.) }}$, the logarithmic term $\ln \mu^{2} / m_{e}^{2}$ can therefore be dropped out of Equation (81) without prejudice. As has been known for a long time, it is only when one is interested in a physical process such as the one depicted in Figure 8 that the intermediate logarithmic singularity of $\ln \mu^{2} / m_{e}^{2}$ is retained in a first step, and then consistently replaced by a regular term of $\ln E_{l} / m_{e}^{2}$, where $E_{l}$ stands 
for a parameter relevant to the case considered, such as an energy resolution, $E_{l}$, in a measure of the real (cut) photon of Figure 8 [24].

(ii) In the literature unfortunately, the general zero-temperature expression of $\Gamma_{\mu}^{\text {ren. }}\left(Q, Q^{\prime}\right)$ does not seem to exist in closed form [25], and Equation (65) is used instead. Accordingly, the polarisation tensor $\delta \Pi_{\mu \nu}^{(3 ; \text { ren.) }}$ of Equation (79) is not the full one, but a part of it.

The situation can therefore be summarised as follows:

- At soft momenta, $k \sim e T$, the cut photon propagator $D_{\mu \nu}^{+}$of Equation (4) receives one-loop HTL contributions, ${ }^{(1)} D_{\mu v}^{+} \equiv{ }^{\star} D_{\mu v}^{+}$, on the same order of magnitude as the cut bare one, evaluated at the same soft scale.

- At ultra-soft momenta, $k \sim e^{2} T$, the same applies also to ${ }^{(2)} D_{\mu v}^{+}$, or equivalently to the spectral density (58) in the case of our illustration. To be complete, however, the ultra-soft scale would require the consideration of an infinite number of loop diagrams. In this diagrammatic analysis, this is displayed by Equation (59) which corresponds to the three-loop topology of Figure 4, while many more diagrams are known to contribute at this order as well, with contributions of form,

$$
\delta \Pi_{L}^{(n ; \text { ren. })} \sim K^{2}\left[\frac{e^{2} T}{k}\right]^{n} \delta \mathcal{F}_{L}^{(n ; \text { ren. })}\left(k_{0}, k\right), \quad n \geq 3 .
$$

Such a case is not easily dealt with diagrammatically, but, hopefully, the semi-classical approaches [19] may offer a sufficiently controlled alternative.

- This is no longer possible at the hyper-soft momentum scale $k \sim e^{3} T$, where the topology of Figure 6 is able to generate a ${ }^{(3)} D_{\mu v}^{+}$, equivalently a ${ }^{(3)} \rho_{L}$ spectral density,

$$
{ }^{(3)} \rho_{L}\left(k_{0}, k, T\right)=2 \operatorname{Im} \frac{1}{k^{2}+m_{3}^{2} \bar{F}_{1}\left(K^{2} / m_{e}^{2}\right)\left[\left(k_{0} / k\right) Q_{0}\left(k_{0} / k\right)\right]+i \varepsilon} ，
$$

which, over this hyper-soft momentum scale, again is on the same order of magnitude as is the bare one evaluated on the same hyper-soft momentum scale. In deriving Equation (84), Equation (80) is used to identify the constant $m_{3}^{2}$ as

$$
m_{3}^{2}=C^{(3)}\left(e^{3} T\right)^{2}, \quad C^{(3)}=\frac{C^{\text {norm. }}}{12 \pi^{2}}\left[\left(1-\frac{1}{8 \pi}\right)\left(\int_{0}^{\infty} \frac{\mathrm{d} x}{x} \tanh x\right)^{\text {ren. }}+\int_{0}^{\infty} \frac{\mathrm{d} x}{\cosh ^{2} x}\right],
$$

and $\bar{F}_{1}\left(K^{2}\right)$ stands for the expression,

$$
\bar{F}_{1}\left(K^{2} / m_{e}^{2}\right)=1-\theta \operatorname{coth} \theta+2 \operatorname{coth} \theta \int_{0}^{\theta / 2} \mathrm{~d} \varphi \varphi \tanh \varphi+\frac{\theta}{4} \tanh \frac{\theta}{2},
$$

where it is reminded that $\theta$ parametrizes the ratio $K^{2} / m_{e}^{2}$, according to the relation $\sinh ^{2}(\theta / 2)=-K^{2} / 4 m_{e}^{2}$. A plot of $\bar{F}_{1}\left(K^{2} / m_{e}^{2}\right)$ is found in Figure 9.

- In this last case, incompleteness comes from the use of Equation (65) instead of the full $\Gamma_{\mu}^{\text {ren. }}\left(Q, Q^{\prime}\right)$, as stated in remark (i) above. Unless explicitly demonstrated, it is not excluded either that higher loop diagrams contribute at the same hyper-soft scale. Completeness in this diagrammatic analysis is again extremely difficult to control [26].

- The point is therefore that the so far controlled situations of soft and ulta-soft scales doesn't seem to exhaust all of the invariant photonic fluctuations contributing the leading order damping rate calculation, and that the contribution completeness to $\gamma(E, p, T)$ of the hyper-soft scale is not under full control; not to speak of other even softer invariant fluctuations, such as those arising at momentum scale $e^{4} T$, not dealt with in the present paper. 


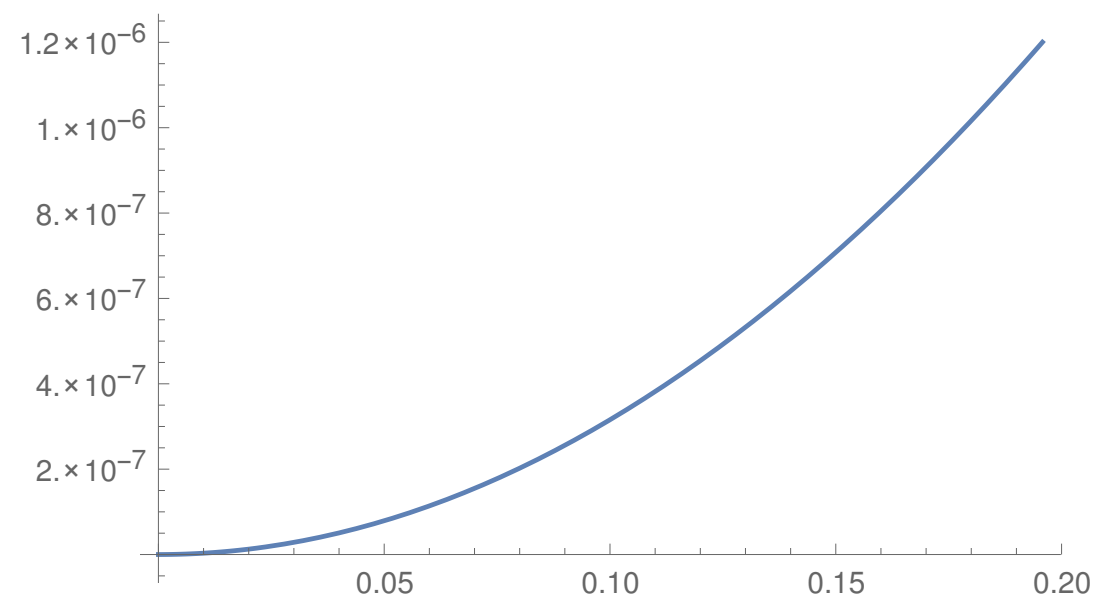

Figure 9. Variations of $\overline{F_{1}}(\theta)$, at $T=100 \mathrm{MeV}, m_{e}=0.51 \mathrm{MeV}$, for $K$ - momenta of order $e^{3} T$.

- Now, there is more to it. Due to the final $k$-momentum integration process, normally taken to be the range $0 \leq k \leq k^{\star},[5,13,14]$, there remains the issue of partitioning the integration range into layers of every decreasing momenta, which contribute to the damping rate $\gamma(E, p, T)$ by comparable amounts.

Each invariant fluctuation, occurring at momentum scale $e^{n} T$, is assigned an effective range (a layer thickness, say) of,

$$
e^{\left[n+\frac{1}{2}\right]} T \leq\left|k_{0}\right| \leq k \leq e^{\left[n-\frac{1}{2}\right]} T,
$$

in agreement with a common, still somewhat arbitrary usage. We know of no theoretical basis which could help with defining the layers in Equation (87) with accuracy [5]. As sketched in Appendix A, a dramatic consequence of this is that even if, at any momentum scale $e^{n} T$, each of the contributing invariant fluctuations yielded a perfectly controlled, complete ${ }^{(n)} D_{\mu \nu}^{+}$pieces (equivalently, a related ${ }^{(n)} \rho_{L}$ spectral densities), their leading order respective contributions to the damping rate, $\delta_{n} \gamma_{L}(E, p, T)$ say, would read typically as,

$$
\delta_{n} \gamma_{L}(E, p, T)=\frac{e^{2} T}{2 \pi} \mathcal{O}_{n}(1), \quad n=1,2,3, \ldots,
$$

where the flexibility in the way Equation (87) can be decided plagues the various values the constants $\mathcal{O}_{n}(1)$ take.

This situation looks suggestive of two major points. First, several gauge-invariant quantum fluctuations of the internal photonic line, taking place at different momentum scales, may contribute at zeroth order to the fermionic damping rate, keeping aside the issue of sub-leading order corrections. Second, completeness of the leading order fermionic damping rate is out of reach in a (perturbative) diagrammatic approach.

A by-product of this result is that the famous infrared divergence, plaguing the rapid fermion damping rate by its mass shell, (9), is an artefact of extending the $k$-integration naively down to zero,

$$
{ }^{\star} \gamma(E, p) \simeq \lim _{v=1} \frac{e^{2} T}{2 \pi} \int_{E|1-v| / 2}^{k^{\star}} \frac{\mathrm{d} k}{k}, \quad k^{\star}=e^{\left[1-\frac{1}{2}\right]} T .
$$

Namely, because of the existence of softer invariant fluctuations contributing at the same order of magnitude (see Appendix A), the HTL effective perturbation theory cannot be extended down to $k=0$, but only to some lower limit, such as $k_{\min }=e^{\left[1+\frac{1}{2}\right]} T$, to comply with Equation (87). Again, no real infrared singularity problem, but definitely something else to be discussed in Section 7. 


\section{Transverse Degrees}

So far, contributions from transverse degrees have been ignored. As displayed in Equation (7) though, the contributions attached to $\delta \Pi_{T}^{(3 ; \text { ren. })}\left(k_{0}, k, T\right) \equiv \Pi_{x x}^{(3 ; \text { ren. })}\left(k_{0}, k, T\right),[5]$, should be considered as well, in the same limit of $k_{\mu} / q \ll 1$ and same topology (Figure 6). Several cases are possible. The transverse contributions are ultra-soft ones, scaling like in Equation (55) and nothing is changed of the previous sections, but for the fact that new contributions to the ultra-soft scale are identified. The transverse contributions are sub-leading with respect to the longitudinal ones (79), so that they can be ignored. Transverse contributions annihilate the longitudinal ones and the matter of previous Section 5 fades away. This is accordingly the point to be analysed.

As calculations display, however, $\delta \Pi_{T}^{(3)}\left(k_{0}, k, T\right)$ supplants $\delta \Pi_{L}^{(3)}\left(k_{0}, k, T\right)$ by a large factor of $(T / k)^{2}$, doesn't call for resummation and does not annihilate longitudinal contributions to the rapid fermion damping rate $\gamma(E, p, T)$. This is seen as follows.

Getting back to the diagram of Figure 6, two Traces are to be considered, which are (see Equation (66)),

$$
2 m^{2} F_{1}\left(K^{2}\right) \operatorname{Tr}\left(\gamma^{x} Q \gamma^{x} Q^{\prime}\right)-F_{1}\left(K^{2}\right) Q^{2} \operatorname{Tr}\left(\gamma^{x} Z^{H T L}(Q) \gamma^{x} Q^{\prime}\right)
$$

Calculations are a bit lengthy but straightforward. Up to a sub-leading contribution of,

$$
-\frac{e^{2} m^{2} F_{1}}{8 \pi^{2}}\left[\frac{K^{2}}{4 T^{2}}\right]\left(\int_{0}^{\infty} \frac{\mathrm{d} x}{x} \frac{\mathrm{d}}{\mathrm{d} x} \frac{1}{x} \tanh \frac{x}{2}\right)^{\text {ren. }}
$$

which can obviously be discarded, the first trace of Equation (90) yields

$$
-\frac{e^{2} m^{2} F_{1}}{2 \pi^{2}} \int_{0}^{\infty} \frac{\mathrm{d} x}{\cosh ^{2} x}+\frac{e^{2} m^{2} F_{1}}{2 \pi^{2}} \frac{K^{2}}{k^{2}}\left[\frac{k_{0}}{k} Q_{0}\left(\frac{k_{0}}{k}\right)+Q_{1}\left(\frac{k_{0}}{k}\right)\right]\left(\int_{0}^{\infty} \frac{\mathrm{d} x}{x} \frac{\mathrm{d}}{\mathrm{d} x}\left[x \tanh \frac{x}{2}\right]\right)^{\text {ren. }} .
$$

The second trace of Equation (90) yields

$$
\begin{aligned}
& \frac{e^{2} m^{2} F_{1}}{2 \pi^{2}}\left(\int_{0}^{\infty} \frac{\mathrm{d} x}{x} \tanh x\right)^{\text {ren. }} \\
& +\frac{e^{2} m^{2} F_{1}}{4 \pi^{2}}\left[\frac{1}{2} \ln \frac{2}{\delta}-1\right] \frac{K^{2}}{k^{2}}\left[\frac{k_{0}}{k} Q_{0}\left(\frac{k_{0}}{k}\right)+Q_{1}\left(\frac{k_{0}}{k}\right)\right]\left(\int_{0}^{\infty} \frac{\mathrm{d} x}{x} \tanh x\right)^{\text {ren. }},
\end{aligned}
$$

where again, $\delta=\left(q-q_{0}\right) / q$ regulates the mass singularities which appear at the boundaries of the integration range $-q \leq q_{0} \leq q$. As usual, these singularities are exactly compensated for by those coming from taking the $q_{0}$-discontinuity of the HTL-self energy (19),

$$
\operatorname{disc}_{q_{0}} \Sigma^{x}(Q)=-\sin \theta \cos \varphi \frac{m^{2}}{q} \frac{q_{0}}{2 q} i \pi \Theta\left(-Q^{2}\right) .
$$

In effect, integrating out the angular dependences, one obtains

$$
\begin{array}{r}
-\frac{e^{2} m^{2} F_{1}}{4 \pi^{2}} \int_{0}^{\infty} q \mathrm{~d} q \int_{-q}^{+q} \mathrm{~d} q_{0} \tanh \frac{q_{0}}{2 T}\left(\frac{1}{q_{0}-q}+\frac{1}{q_{0}+q}\right) \\
\left\{\frac{Q^{2}+K^{2}+2 k_{0} q_{0}}{2 k^{2} q^{2}}-\frac{1}{2 k q}\left(1-\left[\frac{Q^{2}+K^{2}+2 k_{0} q_{0}}{2 k q}\right]^{2}\right) \ln \frac{Q^{2}+K^{2}+2 k_{0} q_{0}-2 k q}{Q^{2}+K^{2}+2 k_{0} q_{0}+2 k q}\right\} .
\end{array}
$$

The first term in the second line of Equation (95) is made out of three contributions, attached to $Q^{2}, K^{2}$ and $2 k_{0} q_{0}$, and only the two first ones contribute thanks to the according $k_{0}$-parities. The term $Q^{2}$ gives a real valued contribution,

$$
-\frac{e^{2} m^{2} F_{1}}{2 \pi^{2}}\left[\frac{T}{k}\right]^{2}\left(\int_{0}^{\infty} \frac{\mathrm{d} x}{x} \int_{0}^{x} \mathrm{~d} y y \tanh \frac{y}{2}\right)^{\text {ren. }}
$$


The term $K^{2}$ is responsible for a mass singularity,

$$
-\frac{e^{2} m^{2} F_{1}}{4 \pi^{2}} \frac{K^{2}}{k^{2}}\left[\ln \frac{\delta}{2}\right]\left(\int_{0}^{\infty} \frac{\mathrm{d} x}{x} \tanh x\right)^{\text {ren. }}
$$

and a regular null expression,

$$
-\frac{e^{2} m^{2} F_{1}}{8 \pi^{2}} \frac{K^{2}}{k^{2}} \times \int_{0}^{\infty} \frac{\mathrm{d} x}{x} \int_{-1}^{+1} \frac{\mathrm{d} y}{y-1}\left[y \tanh \frac{x y}{2}-\tanh \frac{x}{2}\right]+\frac{\mathrm{d} y}{y+1}\left[y \tanh \frac{x y}{2}-\tanh \frac{x}{2}\right]=0 .
$$

Note that, for the second term of Equation (93), the piece coming from the constant term of $Q_{1}(x)=x Q_{0}(x)-1$ (i.e., the term -1 ) is exactly cancelled by Equation (97). Since $x Q_{0}(x)+Q_{1}(x)=2 x Q_{0}(x)-1$, the mass singularities attached to the -1 already cancelled out as just stated, it remains to prove that the mass singularities of the second term of Equation (93) are compensated by those of Equation (95). Now, out of the squared bracket in the second line of Equation (95), one has the crossed term

$$
-\frac{1}{2 k q} \times-K^{2} \frac{k_{0} q_{0}}{k^{2} q^{2}}=\frac{1}{2} \frac{K^{2}}{k^{2}} \frac{k_{0}}{k} \frac{q_{0}}{q^{3}}
$$

and the following integration must be performed,

$$
-\frac{e^{2} m^{2} F_{1}}{4 \pi^{2}} \frac{1}{2} \frac{K^{2}}{k^{2}} \frac{k_{0}}{k} \int_{0}^{\infty} \frac{\mathrm{d} q}{q^{2}} \times \int_{-q}^{+q} q_{0} \mathrm{~d} q_{0} \tanh \frac{q_{0}}{2 T}\left(\frac{1}{q_{0}-q}+\frac{1}{q_{0}+q}\right) \ln \frac{Q^{2}+K^{2}+2 k_{0} q_{0}-2 k q}{Q^{2}+K^{2}+2 k_{0} q_{0}+2 k q} .
$$

By the boundaries at $q_{0}= \pm q$, it is then immediate to check that the mass singularities of Equation (100) read as

$$
+\frac{e^{2} m^{2} F_{1}}{4 \pi^{2}} \frac{K^{2}}{k^{2}} \frac{k_{0}}{k} Q_{0}\left(\frac{k_{0}}{k}\right)\left[\ln \frac{\delta}{2}\right]\left(\int_{0}^{\infty} \frac{\mathrm{d} x}{x} \tanh x\right)^{\text {ren. }},
$$

and exactly cancel out the remaining mass singularities of Equation (93).

What remains are contributions of types,

$$
-\frac{e^{2} m^{2} F_{1}}{2 \pi^{2}} \int_{0}^{\infty} \frac{\mathrm{d} x}{\cosh ^{2} x}, \quad-\frac{e^{2} m^{2} F_{1}}{2 \pi^{2}} \frac{K^{2}}{k^{2}} \delta \mathcal{F}_{T}^{(3)}\left(k_{0}, k\right)
$$

which, for the second type, are similar to those of the longitudinal case (80) up to different overall multiplicative constants. The ensuing transverse spectral density would accordingly read as

$$
\begin{aligned}
& { }^{(3)} \rho_{T}\left(k_{0}, k, T\right) \simeq \\
& 2 \operatorname{Im}\left\{\frac{1}{K^{2}}\left[1+\frac{e^{2} m^{2} F_{1}}{2 \pi^{2} k^{2}}\left(\frac{T^{2}}{K^{2}}\left(\int_{0}^{\infty} \frac{\mathrm{d} x}{x} \int_{0}^{x} \mathrm{~d} y y \tanh \frac{y}{2}\right)^{\text {ren. }}+\delta \mathcal{F}_{T}^{(3)}\left(k_{0}, k\right)\right)\right]^{-1}\right\},
\end{aligned}
$$

with, like $\delta \mathcal{F}_{L}^{(3)}\left(k_{0}, k, T\right), \delta \mathcal{F}_{T}^{(3)}\left(k_{0}, k, T\right)$ developing an imaginary part at space-like momenta $K$. Now, whatever $F_{1}$, the situation here differs from that of ${ }^{(3)} \rho_{L}\left(k_{0}, k, T\right)$ in Equation (84) because the real-valued contribution (96) outweighs any other contribution to $\delta \Pi_{T}^{(3)}\left(k_{0}, k, T\right)$ by a large factor of $(T / k)^{2}$. This leads to a serious suppression of the transverse spectral density in comparison to the longitudinal one.

Over momenta of order $e^{3} T$ in effect, orders of magnitude are found to be in the relation,

$$
\mathcal{O}\left({ }^{(3)} \rho_{T}\left(k_{0}, k, T\right)\right)=\mathcal{O}\left(\left(\frac{k^{2}}{T^{2}}\right)^{2}\right) \times \mathcal{O}\left({ }^{(3)} \rho_{L}\left(k_{0}, k, T\right)\right), \quad k \sim e^{3} T .
$$


Contrary to longitudinal contributions, the three-loop transverse contributions of Figure 6 require no further resummation and preserve the peculiarity of the longitudinal ones, as it was necessary to check. Since Equation (90) only differs from the two-loop case in the overall multiplicative form factor $F_{1}\left(K^{2}\right)$, the same relation as (104) applies to the case of Figure 2 as well, with a milder depletion factor since in this case, one has $k \sim e^{2} T$. This is why the transverse contributions were not considered in Sections 3 and 4 .

\section{Discussion}

In real-time formalisms, it has long been suspected, though poorly examined, that higher number of loop calculations could reveal some peculiarities. This was mainly expected of the so-called singularity mixing problem, that is, of some products of $T=0$ ultraviolet and $T \neq 0$-infrared singularities (which are leading over the $T=0$ ones in the large- $T$ limit [27]).

However intricate such a singularity mixing may be, there seems to be nothing really fundamental about it. Decades of efforts devoted to the matter in a large enough variety of cases have always proven that, when properly dealt with, all singularities cancel out in the end. As compared to the zero-temperature case, where theorems can be invoked, at non-zero temperature, patterns of singularity cancellations are only rendered more difficult to encompass into some $T=0$-like general statements.

At the origin of this fate, two main reasons can be invoked. First, this is because of the explicit breaking of Lorentz invariance brought about by the thermodynamical equilibrium. Singularity cancellation patterns are not so constrained as they are in the $T=0$ Lorentz symmetric case. In a second place, as is done throughout the current paper, one keeps analyzing $T \neq 0$ situations in terms of Feynman diagrams while, ...in contrast to vacuum observables, thermal observables are not themselves primitive blocks of Feynman diagrams [11]. According to the observables under consideration, case-by-case treatments are therefore required, which render difficult attempts at generalizations (note that a somewhat related facet of this state of affairs is the one put forth in [28]).

Now, as advertised above and supported by a large number of calculations, singularity cancellations should rather be regarded a technical matter rather than a real issue. This is why the current paper, already technical enough, doesn't pay more attention to the (ultraviolet diverging) constants of Equation (85) or Equation (A5), which a relevant and canonical renormalization procedure will turn into finite numbers.

Rather, it is here posited that the singularity problem (infrared sector in particular) of non-zero temperature quantum field theories may not be that main issue we've long thought it is [5]; and that it has long masked a more fundamental difficulty, as soon as one tries to enforce a perturbative treatment of these theories. The alluded more fundamental difficulty surfaces already at the level of the historical infrared singularity problem of Equation (9) which, as it should be clear now, is artificial and arises from a too liberal continuation of the $k$-integration in Equation (9) down to zero within the HTL-resummation program.

The fundamental difficulty could be, instead, that any bare and/or effective perturbative treatment be simply unable to reach a complete answer. The example considered in this paper may be regarded as rather suggestive. The damping rate of a fast moving muon is calculated at leading order, as it travels through a QED (or QCD) plasma in thermal equilibrium. Not only the soft, order $e T$, but ultra-soft, order $e^{2} T$, and hyper-soft, order $e^{3} T$ gauge-invariant quantum fluctuations of the emitted and re-absorbed photon contribute the zeroth order muon damping rate, each. In this latter $e^{3} T$ instance, one observes that the gauge invariant character of the associated fluctuations results from an interplay of a $T \neq 0 H T L$-self energy insertion with, at $T=0$, the gauge invariance of the one-loop renormalized vertex; that is, results from a peculiarity of a calculation performed at a high enough number of loops (of course, such a mixing of $T \neq 0$ with $T=0$ parts wouldn't come about in a Matsubara imaginary time formalism, but while this formalism is more fitted to the calculations of static quantities, it is not deprived of analogous difficulties either [29]). 
Now, aside from another quite obvious case at momentum scale $e^{4} T$, not addressed in the present paper, possible softer and softer gauge invariant fluctuations could contribute also on the same order. More precisely, on the basis of diagrammatics, it may be extremely difficult, if even doable, to control the possible number of similar gauge invariant fluctuations. By gauge invariant, what is understood is that no gauge dependence other than that of the original bare photonic line is retained within the higher number of loop fluctuations of this line.

As a result, even the zeroth order estimate of a physical quantity could be out of reach of Perturbation Theory bare and/or effective, not even speaking of further sub-leading corrections. More than the issue of singularity cancellations, which is of a technical nature, this difficulty could constitute a serious obstruction of principle to the application of Perturbation Theory in finite-temperature gauge-field theory.

It is instructive to compare this point of view to the ones suggested in the $C^{\star}$-algebraic analysis of [11]. In [11], in effect, the so-called GNS-construction is used to build the thermal representation space of states $\mathcal{H}_{\omega}$, and no state vector can be found in it out of which to devise a $T=0$-like Perturbation Theory. A perturbativization of the situation is therefore proposed, based on eigen-states of $\mathcal{H}_{\omega}$ which may be thought of in analogy to $T=0$ unstable particle states. To some extent, these states may be viewed as corresponding to the quasi-particle excitations of the plasma, as long identified within the $H T L$-resummation program at scale $e T$, and later on within the ultra-soft effective theory at scale $e^{2} T$. In itself, using unstable/quasi-particle states instead of stable asymptotic ones doesn't preclude a perturbative approach, in agreement with the perturbativization procedure evoked above, or the example explicitly worked out in [30].

Now, it must also be realized that the point of view advocated in the current paper goes one step farther. Though possibly defined in a gauge invariant way, at least when explicitly constructed, there is no systematic control of the quantum fluctuations which may contribute to a given thermal observable at a given order of approximation; and thus there is no possibility to claim at a complete answer at any order of approximation. In the context of non-zero temperature quantum field theories, perturbative attempts could very well be doomed to failure because of this difficulty in which case the whole idea of diagrammatic perturbation theory at finite temperature may have to be abandoned, as quoted in [11]. This is not too surprising in the end if one considers that Feynman diagrams are essentially tied to particle representations, while thermal representations of interacting relativistic field theories have long been known to possess no particle structure [11,31].

There is more on this: for the sake of illustration, the example of QED was used in the current paper and the case of QCD claimed to be the same up to constant redefinitions, like Equation (14). Now, the point is that the $\alpha \simeq 1 / 137$ expansion parameter of QED has no equivalent in QCD. That is, if the QED coupling may be considered small enough to devise clear-cut separated momentum scales, such as $e T, e^{2} T, e^{3} T$, etc.., it is not so in QCD where a clear-cut separation of momentum scales $T$, $g(T) T, g^{2}(T) T, g^{3}(T) T$, etc. is deprived of any physical realisation even at very high $T$, as checked up to $10^{25-30} \mathrm{Tc}$ in the pure Yang-Mills case [32]. A consequence is that, as noticed by J.P. Blaizot in 1999, a condition necessary for a consistent implementation of the renormalisation group $a$ la Wilson fails to be met. The assumption on which one can rely in the case of QED doesn't extend to the case of QCD where, at best, it defines an academic game.

At the very least, it appears that high-temperature quantum field theories call for non-perturbative methods [33]. A new $T \neq 0$ formalism was born very recently [34], which, by construction, implementing temperature by compactifying a spatial rather than the time direction, could perhaps disentangle the infrared sector from the high temperature limit of covariant (not invariant!) quantum field theories and avoid, in this way, the infrared enhancement mechanism, (i.e., the enhancement of infrared contributions remaining after infrared singularities cancellations have been taken into account: In our examples, the integration measures $d k / k$ appearing in Equations (89) and (A3) are responsible for this enhancement. They are induced by the high-temperature expansion of Equation (7)). To our knowledge, however, the new formalism has not yet been explored in this respect. 
Here may be the place where to recall another approach, functional and non-perturbative, concerning a similar calculation [35,36], that of the full fermionic 2- point function $S_{F}^{\prime}\left(E, z_{0}\right)$ under conditions identical to those considered in this paper. The initial energy of the fermion is $E$, and $z_{0}=x_{0}-y_{0}$ measures the travelling duration of the energetic fermion of mass $m$ entering at time $x_{0}$ the QED plasma thermalized at temperature $T$. A Bloch-Nordsieck estimate of $S_{F}^{\prime}\left(E, z_{0}\right)$ reads as

$$
\begin{aligned}
& S^{\prime}\left(E, z_{0}\right)=i \frac{2 m}{E}\left\{\frac{1}{2} e^{-i E z_{0}-\frac{A^{2}}{4 E^{2}} z_{0}^{2}}\right. \\
& \left.-e^{-\frac{E}{T}-\frac{A^{2}}{4 E^{2}}\left(z_{0}^{2}-\frac{1}{T^{2}}\right)} \cos \left(\left[E-2 T\left(\frac{A}{2 E T}\right)^{2}\right] z_{0}\right)\right\},
\end{aligned}
$$

where

$$
A^{2}=\frac{4 \alpha}{3 \pi}\left(\vec{p}^{2}\right)^{2}\left(1+\left(\frac{2 \pi T}{p}\right)^{2}\right)-\frac{4 \alpha^{2}}{3 \pi}\left(\vec{p}^{2}\right)^{2} \ln \left(\frac{\vec{p}^{2}}{m^{2}}\right) .
$$

With $\vec{p}=\vec{p}\left(z_{0}\right)=\vec{p}(0) e^{-\Gamma z_{0}}$, and $\lambda_{c}$ the fermionic Compton wavelength, the momentum damping rate is

$$
\Gamma=\frac{2}{\sqrt{3 \pi}} \frac{\alpha c}{\lambda_{c}}\left(\frac{k_{B} T}{m c^{2}}\right)^{2}
$$

It is worth observing the large variety of different regimes and behaviours comprised within this non-perturbative Bloch-Nordsieck estimate of $S_{F}^{\prime}\left(E, z_{0}\right)$, according to the relative values of the involved parameters, and as time goes by. A richness that, at least, the perturbative attempts will have lead one to suspect.

It is worth mentioning also that another alternative to the perturbative loop expansion can be found within the approach developed in [37], starting out with an explicit construction of the thermal ground state in terms of certain (anti) self-dual Yang-Mills configurations known as Harrington-Shepard (anti) calorons.

Author Contributions: All three authors took equal contributions to the paper.

Funding: The authors acknowledge financial support by Deutsche Forschungsgemeinschaft within the funding programme Open Access Publishing, by the Baden-Württemberg Ministry of Science, Research and the Arts and by Ruprecht-Karls-Universität Heidelberg. R.H. received funding of a visiting (DR2) position by CNRS at the 'Insitut de Physique de Nice' in the summer of 2016, when parts of this work were carried out. I.B. is supported by the International Max Planck research school for precision tests of fundamental symmetries (IMPRS-PTFS) and received support by ITP of Heidelberg University for a stay at the 'Institut de Physique de Nice' in summer 2016.

Acknowledgments: I.B. is enrolled at Heidelberg University.

Conflicts of Interest: The authors declare no conflict of interests.

\section{Appendix A}

In QED, the non-vanishing spectral density of Equation (84),

$$
{ }^{(3)} \rho_{L}\left(k_{0}, k, T\right)=2 \operatorname{Im} \frac{1}{k^{2}+m_{3}^{2} \bar{F}_{1}\left(K^{2} / m_{e}^{2}\right) \frac{k_{0}}{k} Q_{0}\left(\frac{k_{0}}{k}\right)+i \varepsilon},
$$

where $m_{3}^{2}$ is defined in Equation (85), can be integrated out by using the small $\theta$ limit of Equation (82), that is $\bar{F}_{1} \simeq K^{2} / 8 m_{e}^{2}$, giving for the longitudinal fermionic damping rate an amount,

$$
\delta_{3} \gamma_{L}(E, \vec{p}, T)=\frac{e^{2} T}{2 \pi} \int_{e^{7 / 2} T}^{e^{5 / 2} T} k \mathrm{~d} k \int_{-k}^{+k} \frac{\mathrm{d} k_{0}}{k_{0}} \operatorname{disc}_{k_{0}} \frac{1}{k^{2}+m_{3}^{2} \frac{k_{0}^{2}-k^{2}}{8 m_{e}^{2}} \frac{k_{0}}{k} Q_{0}\left(\frac{k_{0}}{k}\right)+i \varepsilon} ，
$$


where Equation (87) is used. Integration on $k_{0}$ is easily carried out by using an energy sum rule [5] and the result is

$$
\delta_{3} \gamma_{L}(E, \vec{p}, T)=\frac{e^{2} T}{2 \pi} \int_{e^{7 / 2} T}^{e^{5 / 2} T} \frac{\mathrm{d} k}{k}=\frac{e^{2} T}{2 \pi} \ln \frac{1}{e},
$$

a result which is worth comparing to the one-loop $H T L$ result,

$$
\delta_{1} \gamma_{L}(E, \vec{p}, T)=\frac{e^{2} T}{2 \pi}\left(\frac{1}{2} \ln \frac{1}{e}-\frac{1}{2} \ln 3+\mathcal{O}(\sqrt{\alpha})\right)
$$

where Equation (14) has been used, which is $m_{\mathrm{QED}}^{2}=e^{2} T^{2} / 6$. The same result can also be shown to apply to the two-loop case of Section 3, when integrated over its relevant momentum range, $e^{5 / 2} T \leq k \leq e^{3 / 2} T$. Gathering all contributions to $\delta \Pi_{L}^{(2)}\left(k_{0}, k, T\right)$, one finds a result of form (A4) with, instead of $\ln 3$ the term

$$
\ln \frac{1}{72 \pi^{2}}\left(\int_{0}^{\infty} \frac{\mathrm{d} x}{x} \frac{\mathrm{d}}{\mathrm{d} x}\left[x \tanh \frac{x}{2}\right]\right)^{\text {ren. }} .
$$

As claimed in Section 5, Equation (88), these invariant fluctuations, at $n=1,2$ and 3 , generate contributions on the same order of magnitude, but, for the reasons advertised in the main text, none of the constants $\mathcal{O}_{n}(1)$ does enjoy an unequivocal determination even though one would benefit from a complete knowledge of the $\delta \mathcal{F}_{L}^{(n ; \text {;ren. })}\left(k_{0}, k, T\right)$ at each momentum scale $e^{n} T$.

In the case of $Q C D$, the smallness of the ratio $-K^{2} / 4 m_{q}^{2}$ is more questionable for light quarks, and Equation (82) may be no longer appropriate. In this situation, however, it is possible to rely on the bounded, monotonic and constant sign variations of $\bar{F}_{1}$, Equation (86), such as displayed below, and a mean value theorem in order to reach exactly the same conclusions for light quarks.

\section{References}

1. Pisarski, R.D. Resummation and the gluon damping rate in hot QCD. Nucl. Phys. A 1991, 525, 175. [CrossRef]

2. Braaten, E.; Pisarski, R. Resummation and Gauge Invariance of the Gluon Damping Rate in Hot QCD. Phys. Rev. Lett. 1990, 64, 1338. [CrossRef] [PubMed]

3. Braaten, E.; Pisarski, R. Soft Amplitudes in Hot Gauge Theories: A General Analysis. Nucl. Phys. B 1990, 337, 569. [CrossRef]

4. Frenkel, J.; Taylor, J.C. High Temperature Limit of Thermal QCD. Nucl. Phys. B 1990, 334, 199. [CrossRef]

5. Le Bellac, M. Thermal Field Theory; Cambridge University Press: Cambridge, UK, 1996.

6. Pisarski, R.D. Scattering Amplitudes in Hot Gauge Theories. Phys. Rev. Lett. 1989, 63, 1129. [CrossRef]

7. Peigné, S.; Pilon, E.; Schiff, D. The Heavy fermion damping rate puzzle. Z. Phys. C 1993, 60, 455. [CrossRef]

8. Grandou, T.J. Proof of a mass singularity free property in high temperature QCD. Math. Phys. 2003, 44, 611. [CrossRef]

9. Grandou, T.J. Angular intricacies in hot gauge field theories. Math. Phys. 2004, 45, 4754. [CrossRef]

10. Bouakaz, K.; Grandou, T. Revisitation of the original hot QCD collinear singularity problem. Quantum Matter 2013, 2, 1. [CrossRef]

11. Landsman, N.P. Nonshell Unstable Particles in Thermal Field Theory. Ann. Phys. 1988, 186, 141. [CrossRef]

12. Candelpergher, B.; Grandou, T. On the damping rate of a moving fermion in hot gauge theories. Ann. Phys. 2000, 283, 232. [CrossRef]

13. Blaizot, J.P.; Iancu, E. Lifetimes of quasiparticles and collective excitations in hot QED plasmas. Phys. Rev. D 1997, 55, 973. [CrossRef]

14. Blaizot, J.P.; Iancu, E. The Bloch-Nordsieck propagator at finite temperature. Phys. Rev. D 1997, $56,7877$. [CrossRef]

15. Grandou, T. A remark on the high temperature limit of QCD. Mod. Phys. Lett. A 2010, 25, 2099. [CrossRef]

16. Blaizot, J.P.; Ollitrault, J.-Y.; Iancu, E. Quark-Gluon Plasma 2; Hwa, R.C., Ed.; World Scientific: Singapore, 1996.

17. Gradshteyn, I.S.; Ryzhik, I.M. Tables of Integrals, Series and Products; Academic Press: San Diego, CA, USA, 1994.

18. Blaizot, J.P.; Iancu, E. Ultrasoft amplitudes in hot QCD. Nucl. Phys. B 2000, 570, 326. [CrossRef] 
19. Bödeker, D. Diagrammatic approach to soft nonAbelian dynamics at high temperature. Nucl. Phys. B 2000, 566, 402. [CrossRef]

20. Rebhan, A.K. Comment on 'high temperature fermion propagator: Resummation and gauge dependence of the damping rate'. Phys. Rev. D 1992, 46, 4779. [CrossRef]

21. Adkins, G.S. One Loop Renormalization of Coulomb Gauge QED. Phys. Rev. D 1983, 27, 1814. [CrossRef]

22. Itzykson, C.; Zuber, J.B. Quantum Field Theory; McGraw-Hill: New York, NY, USA, 1980; p. 335.

23. Muta, T. Foundations of Quantum Chromodynamics; World Scientific: Singapore, 1987.

24. Peskin, M.E.; Schroeder, D.V. Quantum Field Theory; Westview Press: Boulder, CO, USA, 1995; pp. $199-202$.

25. Karplus, R.; Kroll, N.M. Fourth-Order Corrections in Quantum Electrodynamics and the Magnetic Moment of the Electron. Phys. Rev. 1950, 77, 536. [CrossRef]

26. ECT ${ }^{\star}$ Workshop. Mathematical aspects of hadron physics, Trento, Italy, 8-12 October 2012. Available online: https:/ / folk.ntnu.no/kurusche/Trento-ECT-Trento-Title-Abstracts.pdf (accessed on 11 March 2019).

27. Grandou, T.; Reynaud, P. On the thermal effective expansion of a scalar theory. Nucl. Phys. B 1997, 486, 164. [CrossRef]

28. Evans, T.S. What is being calculated with Thermal Field Theory? arXiv 2011, arXiv:hep-ph/9404262.

29. Linde, A.D. Infrared Problem in Thermodynamics of the Yang-Mills Gas. Phys. Lett. B 1980, 96, 289. [CrossRef]

30. Altherr, T.; Grandou, T.; Pisarski, R.D. Thermal instability in $\left(\phi^{3}\right)_{6}$. Phys. Lett. B 1991, 271, 183. [CrossRef]

31. Narnhofer, H.; Requardt, M.; Thirring, W. Quasiparticles At Finite Temperatures. Commun. Math. Phys. 1983, 92, 247. [CrossRef]

32. Akerlund, O.; de Forcrand, P. Scale hierarchy in high-temperature QCD. arXiv 2013, arXiv:1312.1543v1.

33. Lowdon, P. Probing the analytic structure of QCD propagators. In Proceedings of the ICNFP, Kolymbari, Greece, 4-12 July 2018.

34. Reinhardt, H. Hamiltonian finite-temperature quantum field theory from its vacuum on partially compactified space. arXiv 2016, arXiv:1604.06273.

35. Fried, H.M.; Grandou, T.; Sheu, Y.-M. Bloch-Nordsieck Estimates of High-Temperature QED. Phys. Rev. D 2008, 77, 105027. [CrossRef]

36. Candelpergher, B.; Fried, H.M.; Grandou, T. Bloch-Nordsieck estimates of a high-temperature scalar field theory. Int. J. Mod. Phys. 2005, A20, 7525. [CrossRef]

37. Hofmann, R. The Thermodynamics of Quantum Yang-Mills Theory: Theory and Application, 2nd ed.; World Scientific: Singapore, 2016, ISBN 13978-981-4329-04-0.

(C) 2019 by the authors. Licensee MDPI, Basel, Switzerland. This article is an open access article distributed under the terms and conditions of the Creative Commons Attribution (CC BY) license (http://creativecommons.org/licenses/by/4.0/). 